\title{
Magnetic Particle Biosensors
}

\section{Alexander Hrin and Paivo Kinnunen}

\section{Table of Contents}

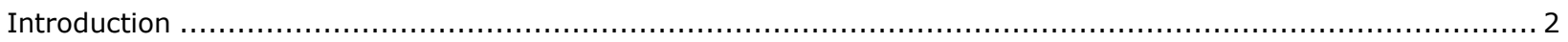

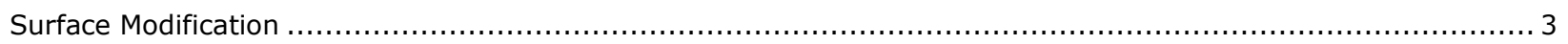

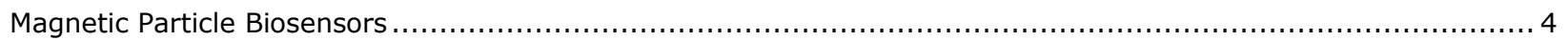

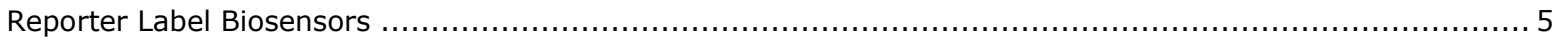

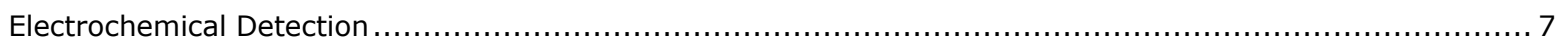

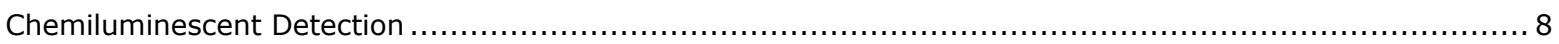

Fluorescent Detection

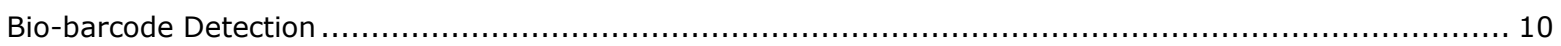

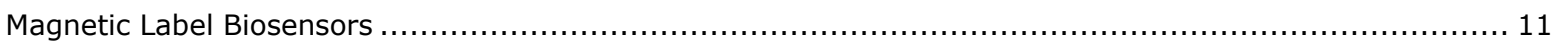

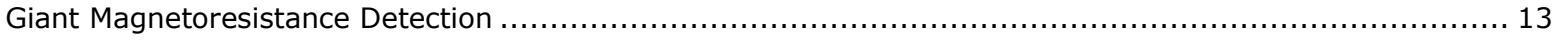

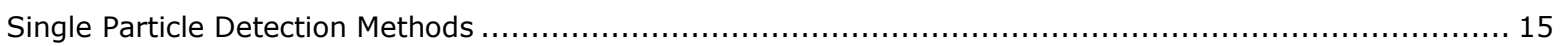

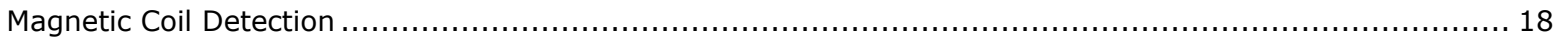

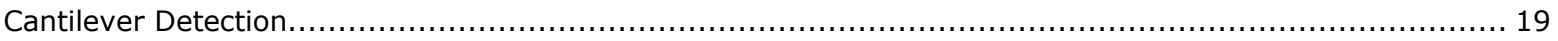

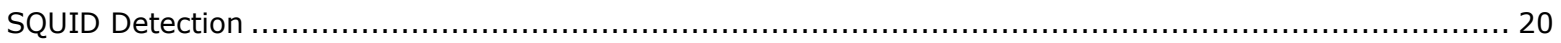

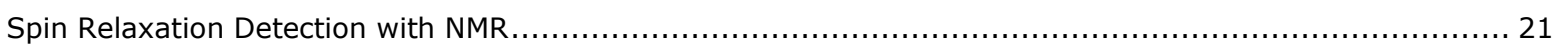

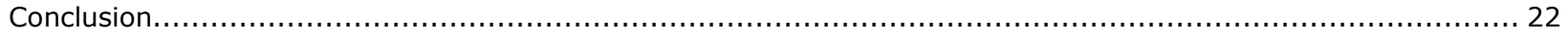

Asynchronous magnetic bead rotation (AMBR) biosensors and biomedical applications ................................. 22

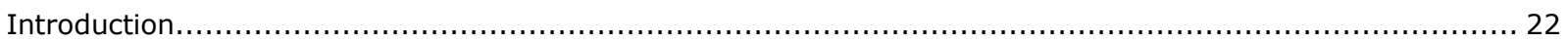

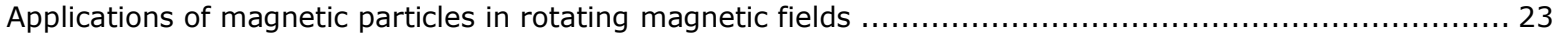

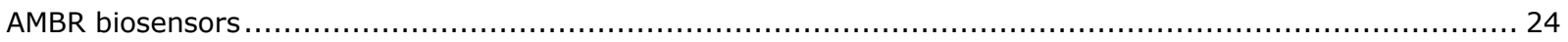

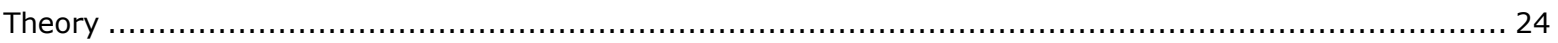

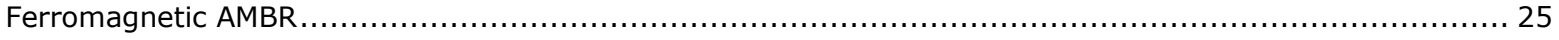

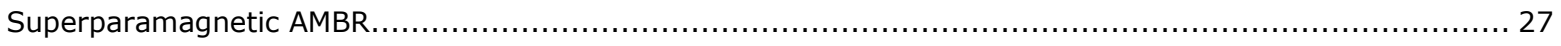

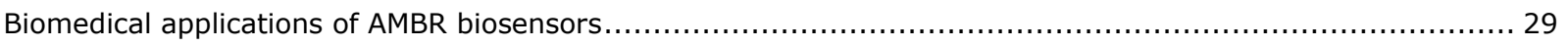

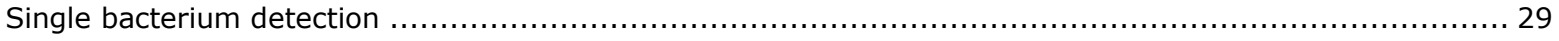

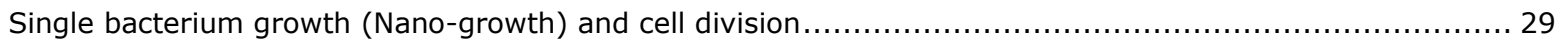

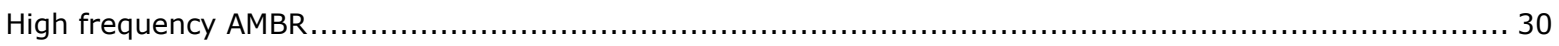

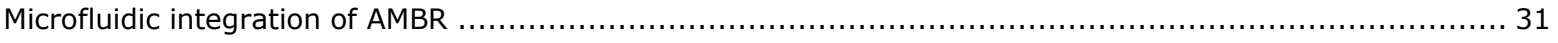

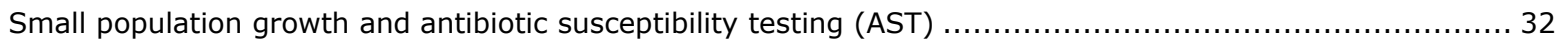

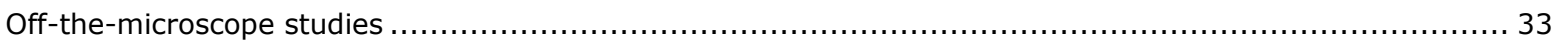

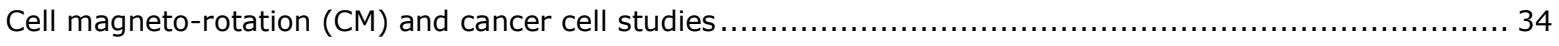

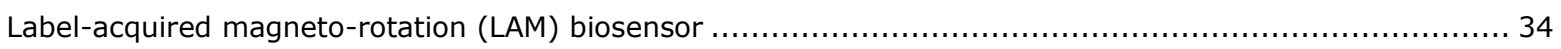

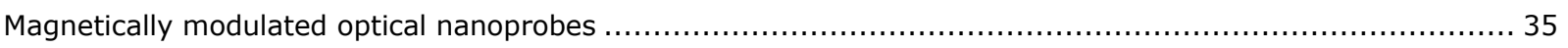

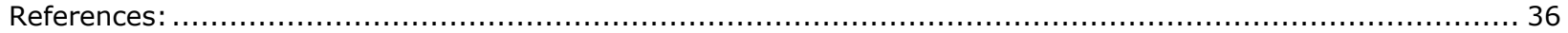




\section{Introduction}

Biosensors are a set of relatively new technologies that have been used to detect the presence of various biological agents. Development of more effective biosensors has been a major area of research with the goal of obtaining methods for early detection of cancers, infections and other diseases we are confronted with today. A few important parameters to consider when designing a biosensor are: speed (how quickly the biosensor assay can be performed), sensitivity (the minimum amount of biological target that can be detected), specificity (how well the biosensor detects only the target analyte), simplicity (the ease with which the assay can be performed), dynamic range (the range of concentrations of the target over which the biosensor is capable of reliably detecting the target), robustness (how well the assay can be reliably performed under varying conditions), amount of sample required (how much of the biological sample of interest is required for use with the assay), and multiplexing (the ability to detect multiple targets with a single assay).

In the past decades, the research involving micro and nanoparticles biosensors has grown by leaps and bounds. As understanding of these systems and the methods of synthesizing magnetic particles have steadily improved, magnetic particles have become more common as a tool in many avenues of this research. Magnetic particles offer a way to increase efficacy of biosensors by increasing the specificity and sensitivity an assay through binding and enrichment of a target analyte. The magnetic particles are able to act as mobile capture vehicles that can bind the targets of interest and be quickly separated or manipulated with the use of an external magnetic field. It has been shown that at low concentration of biological analytes the sensitivity of a biosensor is limited by the binding affinity and binding rates (Sheehan and Whitman 2005). Using a large number of magnetic particles can increase the binding rates and total number of molecules bound to the sensors. When more targets are captured more quickly it makes it simpler for the biosensor to exceed a signal-to-noise ratio $(\mathrm{S} / \mathrm{N})$ of three, which is generally considered to be the limit of detection for biosensors.

Iron oxide magnetic particles-either magnetite $\left(\mathrm{Fe}_{3} \mathrm{O}_{4}\right)$ or maghemite $\left(\gamma-\mathrm{Fe}_{2} \mathrm{O}_{3}\right)$ are among the most prolific in areas of biological research due to their stability and biocompatibility (Tamanaha et al. 2008). These iron oxides display a ferrimagnetic character, meaning that they display a domain structure, but with lower saturation magnetization than related ferromagnetic materials, due to the anti-parallel alignment of adjacent moments. The domain structure of ferrimagnetic materials results in superparamagnetic behavior when 
particle size is smaller than a single domain. A great deal of the work done with magnetic particles takes advantage of the superparamagnetic behavior of very small particles.

Initially, the most prevalent biological application of magnetic particles was in the isolation and separation of desired cellular components (Hsing, Ying, and Wenting 2007). Magnetic separation has been used to isolate nucleic acids (Obata et al. 2002), proteins, enzymes(Safarik and Safarikova 2004), and entire cells (Safarık and Safarıkova 1999) for study. Given that magnetic particles have been successful as a tool for isolating various systems of interest, there has been recent push to discover ways in which the magnetic particles can be used in a way that also facilitates making some kind of measurement of the isolated system of interest. It is in this context that the use of magnetic particles as biosensors has arisen.

\section{Surface Modification}

Before we discuss magnetic particle biosensors, it is worth briefly mentioning of the basic ways in which such synthetic particles are modified to interact with biological systems. In order to have specific, directed interactions with biological components of interest, the surfaces of magnetic particles are modified to facilitate the desired interactions. The first step in modifying the surfaces of magnetic particles is generally to encapsulate the magnetic material in some kind of coating. This encapsulation serves a several purposes, the first of which is to increase the biocompatibility of the particles (Gupta and Gupta 2005). Without a coating of some kind, the particles will often form clusters due to hydrophobic interactions. Once these clusters are formed, they may start exhibiting dipole-dipole interactions, which in turn cause the clusters to become larger, which can be problematic if the particles are to be used as a biosensor. A hydrophilic or amphiphilic coating on the particles will help to prevent this kind of agglomeration. Another potential benefit of using biocompatible shells to encapsulate magnetic particles is the capacity to form micron-sized beads consisting of many superparamagnetic nanoparticles embedded in a polymer matrix. The popular Dynabeads from Invitrogen are precisely this kind of particle. The advantage of these magnetic beads is the fact that they contain a much higher density of magnetic material (and hence they have a faster response to external magnetic fields) while retaining the superparamagnetic character of the nanoparticles (Lea et al. 1988).

Many different materials have been used to provide surface coatings and matrices for magnetic particles. Both synthetic and biopolymers have been commonly used for this purpose. Among the most popular polymeric coatings have been polyethylene glycol (PEG), 
dextran, polyvinyl alcohol (PVA), and polyacylamide, and numerous. Non-polymer coatings, such as stearic acid, silica and gold, have also been used to modify the surfaces of magnetic particles (Gupta et al. 2007). In some cases the encapsulating agents have been directly used as targets for biological interactions. Dextran coatings have been used to bind the biomolecule Concanavlin $A$ in several different experiments demonstrating the efficacy of magnetic particles as biosensors (Kriz, Radevik, and Kriz 1996). Rather than try to find a coating that can also be used as a targeting mechanism, most magnetic particles used in biological systems have the desired targeting ligands or receptor proteins directly attached to the surface of the magnetic particles themselves (Gupta et al. 2007). Among the many different proteins and ligands bound to the surface, two of the most common are biotin and avidin variants. The biotin-avidin bond is among the strongest of biological systems (Weber et al. 1989). Coating the particles with one of these two molecules allows further surface modification of generic sets of particles, for instance, magnetic beads coated with streptavidin can be incubated with any number of biotinylated molecules (antibodies, oligonucleotides) to set the desired targeting mechanism of the magnetic beads. This method of modifying magnetic particles has made for great versatility in their use as biosensors.

\section{Magnetic Particle Biosensors}

As previously mentioned, magnetic particles have been employed as key components in numerous diverse biosensors. While the way in which such particles have been used varies a great deal, magnetic particles have turned into successful biosensors because biological systems of interest do not contain any significant magnetic background that would be affected by the relatively small magnetic fields associated with magnetic particle biosensors (Haun et al. 2010). This means that magnetic particles can easily isolate targeted systems or biomolecules, but more importantly, it means that if measurements associated with the magnetic particles can be made reliably, there is no magnetic signal associated with the biological system that could contaminate the information from the magnetic particles. For this reason, even small signals associated with magnetic particles can reliably detect instances of biological interaction.

Since magnetic particles began to be used as biosensors in the mid 1990s, there have been a wide variety of platforms, molecules, and detection methods used for this purpose (Graham, Ferreira, and Freitas 2004; Haun et al. 2010; Hsing, Ying, and Wenting 2007; Kasatkin, Vasil'eva, and Murav'ev 2010; Katz, Willner, and Wang 2004; Kuramitz 2009; 
Megens and Prins 2005; Tamanaha et al. 2008). While the techniques have been numerous, magnetic particle biosensors generally fall into one of two categories, which will be designated: reporter label, and magnetic label. While this categorization is not perfect, it gives an idea about the different ways in which magnetic particles are used as biosensors.

The reporter label method uses the magnetic particles as a solid phase for capturing the target of interest and then uses some kind of "label" to "report" the presence of the target. For this category, the aspect that changes is the reporter-the system or method that is used to detect the presence of the target. The magnetic label method uses the magnetic particles themselves to report the presence of the target of interest. Generally, with the magnetic label system, the target will bind the magnetic particles to some sort of substrate where they will then be detected. In these systems, it is the method of magnetic particle detection that changes throughout the various techniques. It is worth noting that the ability to disperse magnetic particles in solution, and then rapidly isolate them at a surface or region of interest, is a major factor in their ability to serve as functional biosensors (Graham, Ferreira, and Freitas 2004; Haun et al. 2010; Tamanaha et al. 2008). Next we will turn to the different systems that have been used as reporter labels, or detect the magnetic labels.

\section{Reporter Label Biosensors}

With reporter label biosensors the magnetic particles do not play a direct role in the detection of the target. The essentially common part of all these systems is that the magnetic particles are first used to isolate the target of interest from a background of unwanted components. This might consist of a particular sequence of DNA, a particular protein or antibody, or even entire cells. After binding to the magnetic particles, the target can be isolated by magnetic separation of the particles from the other parts that would otherwise make it difficult to detect the target. What differentiates the various systems of detection is the label that is used to report the presence of the target. The reporter aspect of the sensor is crucial since it is the reporter element that allows the presence of the target of interest to be readily detected. The most common reporting methods are: electrochemical detection, chemiluminescent detection, fluorescent detection, and Biobarcode detection. We'll take a brief look at each of these methods in turn. Table 1 provides an overview of the different kinds of reporter-label techniques, magnetic particles, targets and reported sensitivities from a wide array of literature sources. 
Table 1: A survey of the reporter label methods that have been used to detect biological targets is presented below.

\begin{tabular}{|c|c|c|c|c|}
\hline Method & Magnetic Particles & Target & $\begin{array}{l}\text { Reported } \\
\text { Sensitivity }\end{array}$ & Source \\
\hline $\begin{array}{l}\text { Chemiluminescence } \\
\text { (Ru based tag) }\end{array}$ & Invitrogen $2.8 \mu \mathrm{m}$ & E. Coli 0157 cells & 10 cells & (Shelton and Karns 2001) \\
\hline Electrochemical Stripping (Au nanoparticle tags) & Bangs $0.8 \mu \mathrm{m}$ & DNA oligonucleotide & $1.5 \mathrm{nM}$ & (Wang et al. 2001) \\
\hline $\begin{array}{l}\text { Electrochemical stripping } \\
\text { (Ag enhanced Au tag) }\end{array}$ & Unspecified & DNA oligonucleotide & $32 \mathrm{pM}$ & (Wang, Polsky, and Xu 2001) \\
\hline Silver Deposition & Unspecified & DNA oligonucleotide & $50 \mathrm{fM}$ & (Wang, Xu, and Polsky 2002) \\
\hline Bio-barcode & $\begin{array}{l}1 \mu \mathrm{m} \\
\text { Iron Oxide }\end{array}$ & PSA & $3 \mathrm{aM}$ & (Nam, Thaxton, and Mirkin 2003) \\
\hline $\begin{array}{l}\text { Chemiluminescence } \\
\text { HRP/naphtoquinone tags) }\end{array}$ & Unspecified & DNA oligonucleotide & $10 \mathrm{fM}$ & (Weizmann et al. 2003) \\
\hline Electrochemical Stripping (Au coated Fe particles & Bangs $10 \mu \mathrm{m}$ & DNA oligonucleotide & $50 \mathrm{ng} / \mathrm{ml}$ & (Wang 2003) \\
\hline Bio-barcode & $\begin{array}{l}\text { Polysciences (unspecified } \\
\text { size) }\end{array}$ & DNA oligonucleotide & $500 \mathrm{zM}$ & (Nam, Stoeva, and Mirkin 2004) \\
\hline $\begin{array}{l}\text { Electrochemical Label } \\
\beta \text {-galactoseidase }\end{array}$ & Invitrogen 2.8 um & IgG & $\begin{array}{l}3.5 \mathrm{fmol} \\
(70 \mathrm{pM})\end{array}$ & (Thomas et al. 2004) \\
\hline $\begin{array}{l}\text { Electrochemical Stripping } \\
\text { (ZnS label) }\end{array}$ & Unspecified & DNA oligonucleotide & $250 \mathrm{fM}$ & (Zhu et al. 2004) \\
\hline $\begin{array}{l}\text { Electrochemical stripping } \\
\text { MW CNT W/ Alkaline Phosphatase tags }\end{array}$ & Unspecified & DNA oligonucleotide & $54 \mathrm{am}$ & (Wang, Liu, and Jan 2004) \\
\hline$"$ & $"$ & IgG & $6.4 \mathrm{fM}$ & " \\
\hline $\begin{array}{l}\text { Electrochemical Labels } \\
\text { (multiplexed) }\end{array}$ & Bangs $0.83 \mu \mathrm{m}$ & $\begin{array}{l}\text { B2-microglobulin/ } \\
\text { IgG/BSA/CRP }\end{array}$ & $100 \mathrm{ng} / \mu \mathrm{l}$ & (Liu et al. 2004) \\
\hline Bio-barcode & Invitrogen (Unspecified size) & PSA & $10 \mathrm{fg} / \mathrm{ml}$ & (Bao et al. 2006) \\
\hline Bio-barcode (Fluorescence) & Polysciences $1 \mu \mathrm{m}$ & PSA & $300 \mathrm{aM}$ & (Oh et al. 2006) \\
\hline Bio-barcode & Invitrogen $\mathrm{M}-280$ & $\begin{array}{l}\text { DNA oligonucleotide } \\
\text { (multiplexed) }\end{array}$ & $500 \mathrm{fM}$ & $\begin{array}{l}\text { (Stoeva, Lee, Thaxton et al. } \\
\text { 2006) }\end{array}$ \\
\hline Bio-barcode & Invitrogen $1 \mu \mathrm{m}$ & PSA/ HCG/ AFP (multiplexed) & $170 \mathrm{fM}$ & (Stoeva, Lee, Smith et al. 2006) \\
\hline Direct Oxidation & Invitrogen $2.8 \mu \mathrm{m}$ & DNA oligonucleotide & $20 \mu \mathrm{g} / \mathrm{ml}$ & (Erdem et al. 2006)a \\
\hline Microelectrode capacitance measurement & Invitrogen $2.8 \mu \mathrm{m}$ & Salmonella typhimurium cells & $10 \mathrm{CFU} / \mathrm{ml}$ & (Yang and Li 2006) \\
\hline Bio-barcode & Invitrogen $2.8 \mu \mathrm{m}$ & Genomic DNA & $2.5 \mathrm{fM}$ & (Hill, Vega, and Mirkin 2007) \\
\hline Fluorescent Label (Quantum Dots) & Invitrogen $2.8 \mu \mathrm{m}$ & TNF-a & $6 \mathrm{fM}$ & (Agrawal, Sathe, and Nie 2007) \\
\hline Fluorescent Label (Quantum Dots) & Invitrogen $2.8 \mu \mathrm{m}$ & DNA oligonucleotide & $250 \mathrm{zM}$ & (Liu et al. 2008) \\
\hline $\begin{array}{l}\text { Fluorescent Label } \\
\text { (Quantum Dots) }\end{array}$ & Bangs $8.27 \mu \mathrm{m}$ & DNA oligonucleotide & $50 \mathrm{nM}$ & (Lim et al. 2009) \\
\hline $\begin{array}{l}\text { Chemiluminescence } \\
\text { (AgNO3 + luminol) }\end{array}$ & $\begin{array}{l}\text { Polysciences } \\
\text { (Unspecified size) }\end{array}$ & DNA oligonucleotide & $50 \mathrm{aM}$ & (Cai et al. 2010) \\
\hline Electrochemical Label (Lipase/ferrocene) & Invitrogen $1 \mu \mathrm{m}$ & DNA oligonucleotide & $20 \mathrm{amol}$ & (Ferapontova et al. 2010) \\
\hline $\begin{array}{l}\text { Fluorescent Label } \\
\text { (Quantum Dot) }\end{array}$ & $2.8 \mu \mathrm{m}$ & C-reactive protein & $10 \mathrm{pM}$ & $\begin{array}{l}\text { (Zhu, Duan, and Publicover } \\
\text { 2010) }\end{array}$ \\
\hline $\begin{array}{l}\text { Fluorescent Label } \\
\text { (Quantum Dot) }\end{array}$ & Invitrogen $2.8 \mu \mathrm{m}$ & DNA oligonucleotide & $890 \mathrm{zM}$ & (Kim and Son 2010) \\
\hline $\begin{array}{l}\text { Fluorescent } \\
\text { (OliGreen Dye) }\end{array}$ & $\begin{array}{l}\text { Polysciences } \\
\text { (Unspecified size) }\end{array}$ & Genomic DNA & $180 \mathrm{fM}$ & (Wang et al. 2010) \\
\hline
\end{tabular}




\section{Electrochemical Detection}

Electrochemical detection is among the broadest methods of reporting the presence of a biological target. The essential idea is that the system is set up in such a way that when the magnetic particles bind the target and are brought to an electrode, some kind of electrochemical reaction occurs that would not have happened if no target were present. The systems that employ this scheme are numerous and oftentimes seem to have little in common.

One method of electrochemical detection is by direct oxidation of the target (Erdem et al. 2006; Palecek, Fojta, and Jelen 2002). Generally this method has been used to detect target nucleic acids. The first step is to isolate the target with the magnetic particles. Once the target has been secured, it can be removed from the magnetic particles or left attached depending on the system. The target nucleic acids are placed into conditions that will facilitate the electrochemical oxidation of specific

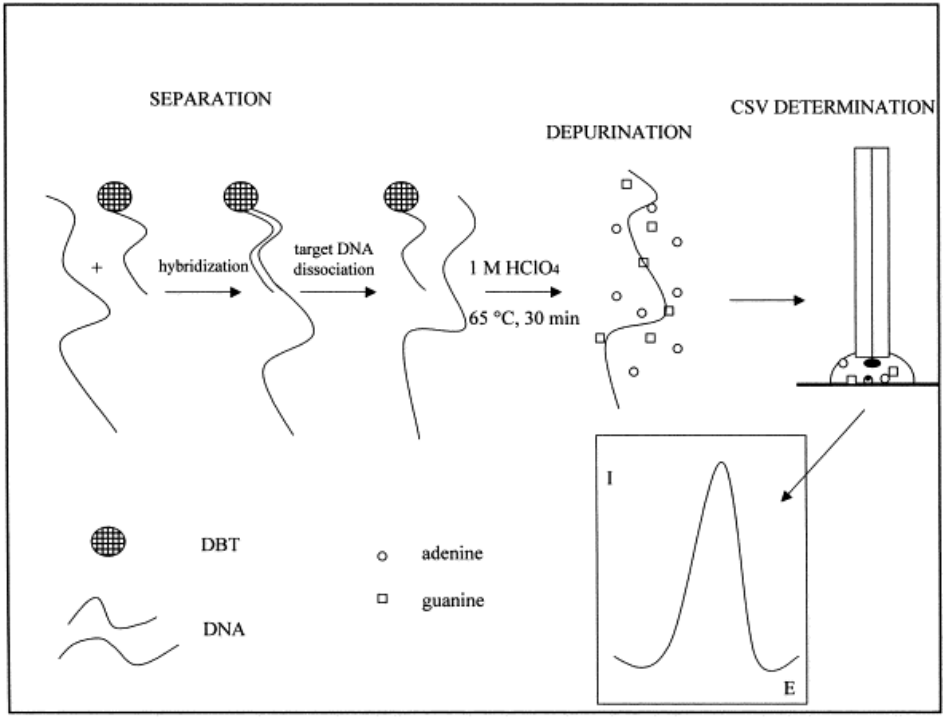

Figure 1: A basic schematic for the direct oxidation process. The magnetic particles are used to capture the DNA. The purine bases are stripped and the resulting signal can be detected electrochemically. bases (purines usually). In this case, the magnitude of the signal from the oxidation will correspond to the amount of target present. This method is often described as "label free" since no other agents are bound to the target to detect the oxidation signal. See Figure 1 for the basic layout of this detection method.

Another method of detection is the electrochemical stripping of metal labels that bind to the target and the magnetic beads (Wang et al. 2001). In this case, the target in fact binds metal labels to the magnetic beads before separation. The number of labels is proportional to the amount of target present. Next the metal label is "stripped" by an acid into metal ions. The stripped metals result in a detectable change in the signal, measured by the electrodes of the system. In addition to simple gold nanoparticles as metal tags, this technique has also been used in conjunction with iron-core gold nanoparticles (Wang 2003), 
semiconductor nanoparticles (Zhu et al. 2004), and gold nanoparticles enhanced by silver deposition (Wang, Polsky, and Xu 2001). This method has also been used to detect multiple targets from a single sample, by relying on the fact that different labels result in different electrochemical signals (Liu et al. 2004). Metal stripping has been used to detect the presence of both nucleic acids and proteins.

A final way of using electrochemical signals to detect the presence of a target nucleic acid or protein is to utilize enzyme labels to start the electrochemical reaction (Gehring et al. 1996). Here we see that the target will attach some sort of active protein label to the magnetic particles (again in a quantity proportional to the amount of target present). Next, the enzymes will be brought to the electrode, where their activity willeither directly or indirectly-cause a change in the detectable electrochemical signal. Many different kinds of enzymes have been used in these techniques, including: $\beta$ -

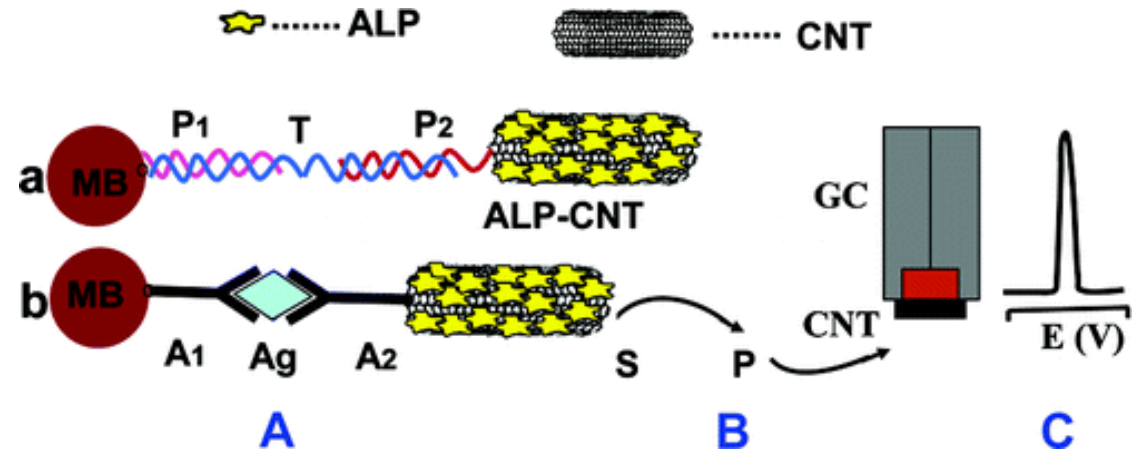
Figure 2: The process by which electrochemical label detection occurs is essentially similar no matter the label being used. Here we have alkaline phosphatase enzymes attached to a carbon nanotube as label. The target binds the label to the magnetic particle, and the electrochemical signal can be detected.

galactoseidase (Thomas et al. 2004), alkaline phosphatase (Wang, Liu, and Jan 2004), and a lipase (Ferapontova et al. 2010). The experimental techniques for electrochemical label detection are similar no matter what label is used. Figure 2 shows an example of a system in which an electrochemical label is used to detect the presence of a target.

Cellular growth has been detected electrochemically by monitoring the changes in impedance of the media as the cells grow and divide (Yang and Li 2006). After isolation and separation of the target cells by magnetic beads, the growth area is essentially inserted between two plates of a capacitor, and the cells are allowed to grow. The growth of the cells causes a change in the dielectric constant, in between the two plates of the capacitor. This change can be detected as a change in the impedance of the connected circuit.

\section{Chemiluminescent Detection}

A method somewhat related to electrochemical detection is chemiluminescent detection. Chemiluminescent detection utilizes an appropriate agent (usually luminol) to detect the 


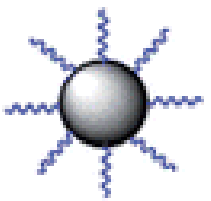

1.Target DNA

2. gold probe

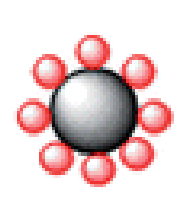

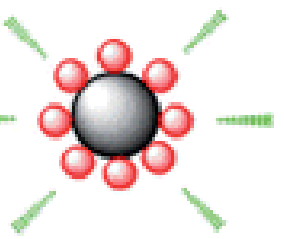

Figure 3: The basic setup for a chemiluminescent label is to use the target to bind a label that can facilitate a chemiluminescent reaction. Without the target there will be no luminescence. presence of the target of interest. In this case, it is a blue glow that is detected when the target of interest is present. However, there are generally

intermediate steps required in order to generate the conditions under which the luminol will exhibit luminescence. It is in this fashion that chemiluminescent detection methods are similar to electrochemical detection methods. For instance, gold nanoparticles are sometimes used as tags that attach to the magnetic beads, in the presence of the target; they are then detected by mixing the isolated magnetic beads with $\mathrm{AgNO}_{3}$ and luminol, causing a luminescence reaction (Cai et al. 2010). Other methods have used enzymes as tags in order to generate the needed oxidizing reactants for luminol to emit its blue glow (Weizmann et al. 2003). In some cases, it's an electrochemical reaction that enables the luminescence (Shelton and Karns 2001), and in this way, chemiluminescence is similar to electrochemical detection in that many similar designs and experimental setups can be used for each. Figure 3 shows the basic setup for a reaction chemiluminescent detection method.

\section{Fluorescent Detection}

Detection of biological analytes by means of fluorescence is one of the most commonly used techniques in the field today (D'Orazio 2003). The use of fluorescence in conjunction with magnetic particle isolation has potential in the sense that the magnetic particles could be used to isolate a small number of

(A)

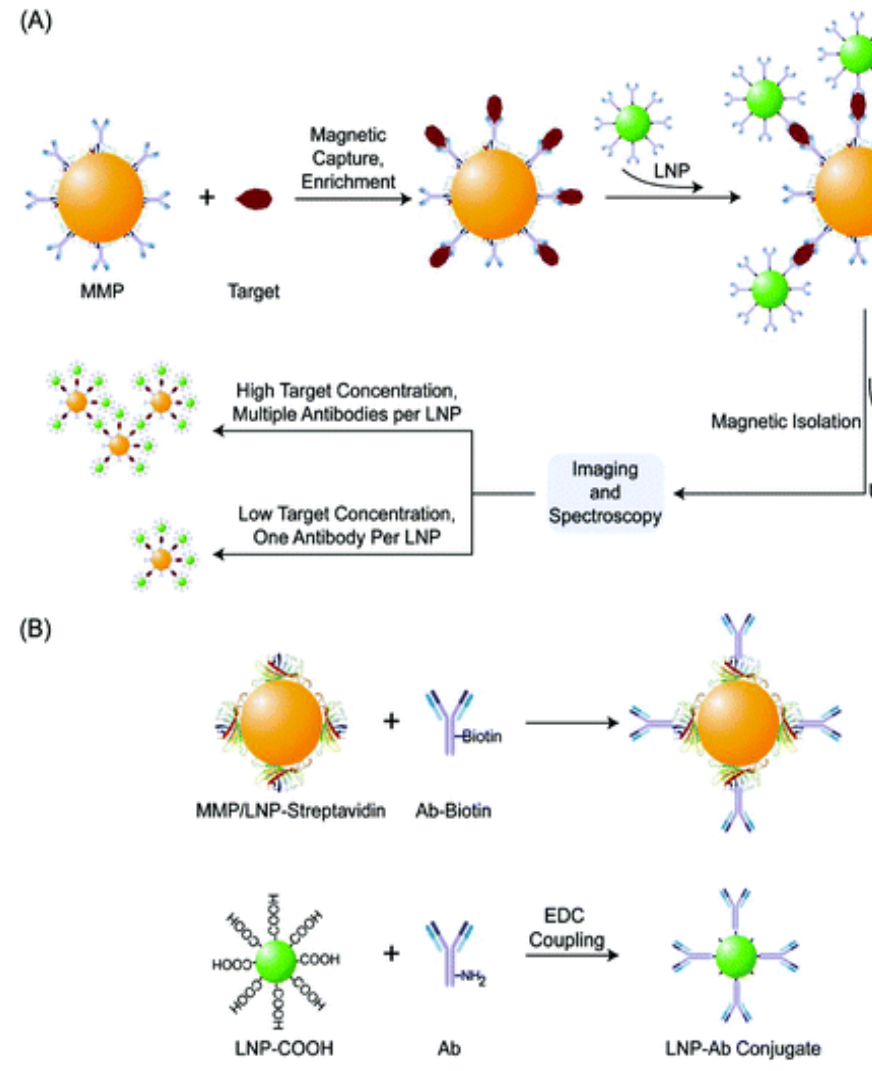

Figure 4: The most common fluorescent labels used to detect biological targets in conjunction with magnetic particles are quantum dots. 
biomolecules on their surface, which could then be detected by means of fluorescence. Again, the idea is to use the target of interest to attach a fluorescent agent to the magnetic particles. Effectively, the magnetic particles are locally enriching the concentration of the target such that the fluorescence is detectable. The target concentration can be quantified because the amount of fluorescence is proportional to the concentration of the target. Many different fluorescent labels have been used in conjunction with magnetic particle detection, including quantum dots (Liu et al. 2008) and DNA-staining dyes (Wang et al. 2010). Figure 4 provides an example of how fluorescent labels have been used to detect target proteins (Agrawal, Sathe, and Nie 2007; Zhu, Duan, and Publicover 2010), but the technique has also been used to detect the presence of nucleic acids (Kim and Son 2010; Lim et al. 2009).

\section{Bio-barcode Detection}

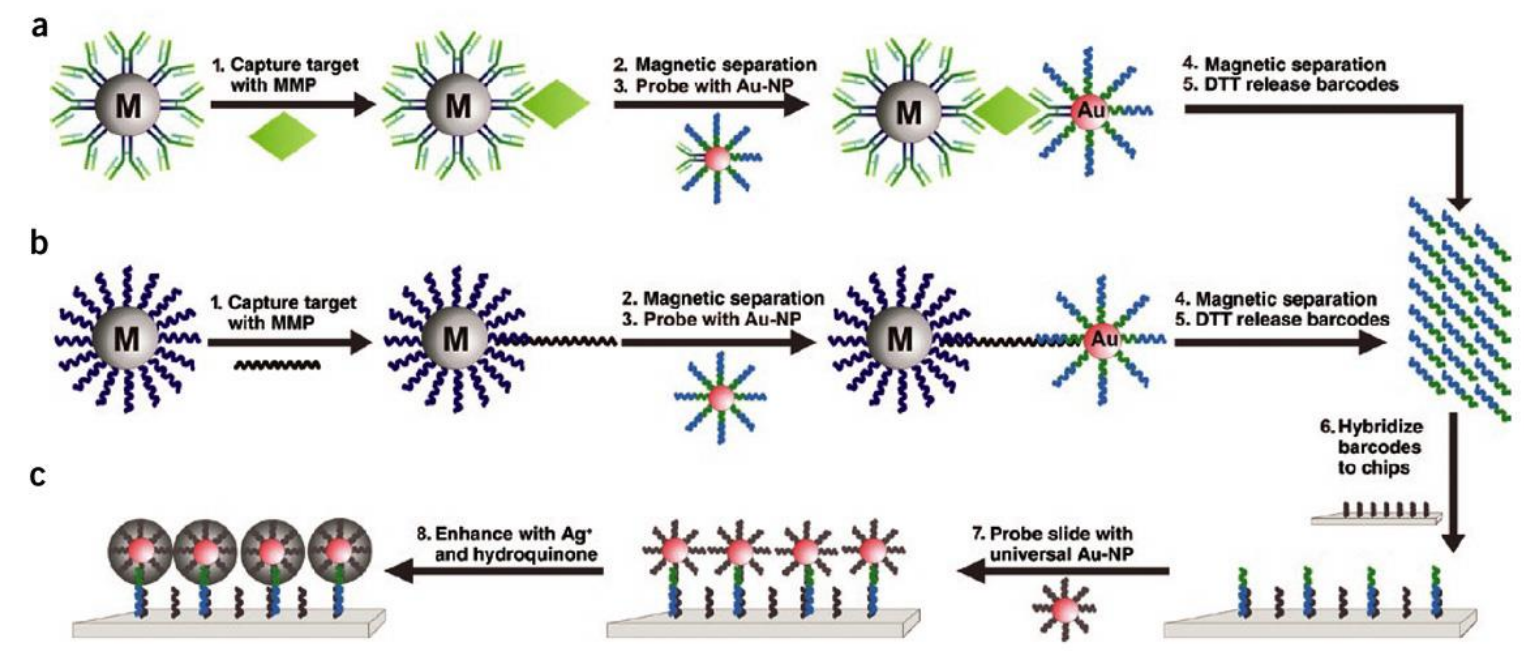

Figure 5: The bio-barcode method has proven both versatile in its ability to detect very minute amounts of both nucleic acids and proteins. The detection method is the same whether or not the setup is detecting DNA or a specific protein.

Bio-barcode detection is a technique developed by the Mirkin group (Hill and Mirkin 2006; Nam, Jang, and Groves 2007). The essential idea behind bio-barcode sensors is that rather than try to detect the target directly, one instead detects a pre-prepared oligonucleotide sequence corresponding to that target. The actual choice of sequence in this case is arbitrary, so long as the barcode can fulfill all the steps needed in the process. The initial bio-barcode research did not use magnetic particles, and relied on the variations in the C-G content of the barcode oligonucleotides to identify different binding analytes (Nam, Park, and Mirkin 2002). The use of magnetic particles made the bio barcode technology far more effective. The magnetic particles are covered with the appropriate detection elements for the target of interest. After capturing and isolating the target with the magnetic particles, 
the system could be incubated with gold nanoparticles that also have the appropriate recognition element attached to the surface, which would form a sandwich complex with the total number of gold nanoparticles captured, in proportion to the amount of target present. In addition to the target recognition element, the gold nanoparticles have a large quantitygenerally around 100x the number of target recognition elements-of barcode DNA on their surface. This means that for every time a gold nanoparticle encounters the target, there will be a large increase in the amount of bio-barcode DNA. Figure 5 shows broadly how the biobarcode method can be used to detect many relevant biological targets.

The barcode DNAs are generally detected using a scanometric method, also developed by the Mirkin group (Taton, Mirkin, and Letsinger 2000). The basic idea is that since the sequence of the barcode DNA is a portion of the complementary sequence, it can be bound to a surface, and gold nanoparticles can be attached to each of the DNA barcodes using the other portion of the complementary sequence. The final step is to catalytically deposit silver onto the gold nanoparticles attached to the surface through the barcode DNA. Once this has been done the surface will be effectively stained a darker color, depending on the concentration of bio-barcode DNA. The extent of this staining corresponds to the amount of target in the initial system of interest.

Another method of detection of the bio-barcodes is with fluorescence (Oh et al. 2006). In this setup, the barcodes are labeled with fluorophores before they are ever attached to the gold nanoparticle probes. Once the target has been isolated by the magnetic particles, the barcodes can be removed and the overall fluorescence of the solution will correspond to the concentration of the barcodes and, by extension, to the concentration of the target.

The bio-barcode method has demonstrated extreme sensitivity, on the order of $10 \mathrm{~s}$ of molecules for some targets (Nam, Thaxton, and Mirkin 2003). It has been successful in detecting low levels of biologically relevant proteins (Bao et al. 2006; Nam, Jang, and Groves 2007), oligonucleotides (Nam, Stoeva, and Mirkin 2004) and genomic DNA (Hill, Vega, and Mirkin 2007) Additionally, bio-barcodes have proven amenable to multiplexing, with the ability to detect multiple targets of both proteins (Stoeva, Lee, Smith et al. 2006) and DNA (Stoeva, Lee, Thaxton et al. 2006).

\section{Magnetic Label Biosensors}

The magnetic label methods of biosensing continue to rely on the capacity of magnetic particles to capture and isolate targets of interest from a mixture of unwanted elements. However, these types of sensors attempt to directly detect the magnetic particles 
Table 2: The various magnetic label methods that have been used to detect biological targets are presented in the table below.

\begin{tabular}{|c|c|c|c|c|}
\hline Method & Magnetic Particles & Target & $\begin{array}{l}\text { Reported } \\
\text { Sensitivity }\end{array}$ & Source \\
\hline Coil/Maxwell Bridge & Dextran Ferrofluid & Glucose & $40 \mathrm{mM}$ & (Kriz, Radevik, and Kriz 1996) \\
\hline SQUID (substrate) & Unspecified & Collagen & $100 \mathrm{pM}$ & (Kotitz et al. 1997) \\
\hline Coil/Maxwell Bridge & Iron Oxide (20-200 nm) Dextran coating & Concanavalin A & $1.5 \mu \mathrm{M}$ & (Kriz, Gehrke, and Kriz 1998) \\
\hline Coil/Resonance & Invitrogen $2.8 \mu \mathrm{m}$ & Human Transferrin & $260 \mathrm{fM}$ & (Richardson, Hawkins, and Luxton 2001) \\
\hline NMR Relaxation & Iron Oxide Dextran Coating $(40-50 \mathrm{~nm})$ & DNA oligonucleotide & $10 \mathrm{pM}$ & (Perez et al. 2002) \\
\hline Giant Magnetoresitance (BARC) & Bangs $350 \mathrm{~nm}$ & DNA Oligonucleotide & $16 \mathrm{pg} / \mathrm{ul}(25 \mathrm{pM})$ & (Schotter 2004) \\
\hline Cantilever & Clontech borosilicate $5 \mu \mathrm{m}$ & Genomic DNA & $71 \mathrm{zM}$ & (Weizmann et al. 2004) \\
\hline " & $"$ & $\begin{array}{l}\text { Telomerase (activity from } \\
\text { cancer cells) }\end{array}$ & 100 cells & $"$ \\
\hline NMR Relaxation & Iron Oxide $40 \mathrm{~nm}$ & $\begin{array}{l}\text { Telomere DNA } \\
\text { (Telomerase activity) }\end{array}$ & $300 \mathrm{amol}$ & (Grimm et al. 2004) \\
\hline Coil/Permeability & $\mathrm{Fe}_{3} \mathrm{O}_{4}$ Dextran Coating $40-110 \mathrm{~nm}$ & C-reactive protein & $0.2 \mu \mathrm{g} / \mathrm{ml}$ & (Kriz et al. 2005) \\
\hline Spin Valve & $250 \mathrm{~nm}$ Nanomag D & DNA oligonucleotide & $10 \mathrm{pM}$ & (Graham et al. 2005) \\
\hline SQUID (substrate) & $\mathrm{Fe}_{3} \mathrm{O}_{4} 25 \mathrm{~nm}$ & IgE & $2 \mathrm{amol}(10 \mathrm{fM})$ & (Enpuku et al. 2005) \\
\hline Hall Probe & Polysciences $2-20 \mathrm{~nm}$ & IgG & $100 \mathrm{pg} / \mathrm{ml}$ & (Aytur et al. 2006) \\
\hline Giant Magnetoresistance (BARC)/ CCD & Invitrogen $2.8 \mu \mathrm{m}$ & DNA Oligonucleotide & $10 \mathrm{fM}$ & (Mulvaney et al. 2007) \\
\hline " & $"$ & Ricin A Chain & $300 \mathrm{fM}$ & $"$ \\
\hline " & $"$ & Staphyloccal enterotoxin B & $300 \mathrm{fM}$ & $"$ \\
\hline CCD & Invitrogen $1 \mu \mathrm{m}$ & $\begin{array}{l}\text { Streptavidin/IgG/ } \\
\text { West Nile Virus Particles }\end{array}$ & $\begin{array}{l}400-800 \\
\text { molecules }\end{array}$ & (Morozov et al. 2007) \\
\hline Coil/Resonance & Seradyne $(0.824 \mu \mathrm{m})$ & Troponin I & $0.5 \mathrm{ng} / \mathrm{ml}$ & (Kiely et al. 2007) \\
\hline SQUID (substrate) & $\mathrm{Fe}_{3} \mathrm{O}_{4}(25 \mathrm{~nm})$ & IgE & $15 \mathrm{amol}$ & (Enpuku et al. 2007) \\
\hline Giant Magnetoresistance (BARC) & MACs $(50 \mathrm{~nm})$ & CEA & $5 \mathrm{fM}$ & (Osterfeld et al. 2008) \\
\hline " & $"$ & G-CSF & $119 \mathrm{fM}$ & $"$ \\
\hline " & $"$ & IL-1a & $53 \mathrm{fM}$ & " \\
\hline " & $"$ & IL-10 & $56 \mathrm{fM}$ & " \\
\hline " & " & IFN- $y$ & $59 \mathrm{fM}$ & $"$ \\
\hline " & $"$ & Lactoferrin & $13 \mathrm{fM}$ & $"$ \\
\hline$"$ & $"$ & TNF-a & $57 \mathrm{fM}$ & $"$ \\
\hline SQUID (no substrate) & Nanomag $(40,130,250 \mathrm{~nm})$ & DNA oligonucleotide & $11 \mathrm{pM}$ & $\begin{array}{l}\text { (Strömberg, Zardán Gómez de la Torre et } \\
\text { al. 2008) }\end{array}$ \\
\hline MTJ & $\mathrm{Fe}_{3} \mathrm{O}_{4}(16 \mathrm{~nm})$ & DNA oligonucleotide & $2.5 \mu \mathrm{M}$ & (Shen et al. 2008) \\
\hline Giant Magnetoresitance (BARC) & Miltenyi (50 nm) & CEA & $50 \mathrm{aM}$ & (Gaster et al. 2009) \\
\hline$C C D$ & Invitrogen $2.8 \mu \mathrm{m}$ & Genomic DNA & $10 \mathrm{fm}$ & (Mulvaney et al. 2009) \\
\hline NMR Relaxation & Iron Oxide $(>100 \mathrm{~nm})$ & $\begin{array}{l}\text { A549 cells (mammalian)/ } \\
\text { MAP cells (bacterial) }\end{array}$ & 10 cells & (Kaittanis, Santra, and Perez 2009) \\
\hline SQUID (no substrate) & $(130,250 \mathrm{~nm})$ & DNA oligonucleotide & $40 \mathrm{pM}$ & (Strömberg et al. 2009) \\
\hline CCD & Invitrogen $1 \mu \mathrm{m}$ & DNA oligonucleotide & $100 \mathrm{aM}$ & (Shlyapnikov et al. 2010) \\
\hline SQUID (substrate) & $\mathrm{Fe}_{3} \mathrm{O}_{4}(25 \mathrm{~nm})$ & IL-8/IgE & $4 \mathrm{amol}$ & (Kuma et al. 2010) \\
\hline " & $"$ & Candida albicans cells & 300 cells & $"$ \\
\hline
\end{tabular}


that have bound the target to their surface. Magnetic label detection methods utilize systems in which the effects of the magnetic particles themselves are measured, rather than the effects of a related reporter. Like with the reporter-label methods, the number of individual techniques reported in the literature is quite numerous, but in a broad sense, the methods of detecting magnetic particles fall into one of the following categories: Anisotropic Magnetic Resistance (AMR); Induction coils; Superconducting Quantum Interference Device (SQUID); and magnetic relaxation using Nuclear Magnetic Resonance (NMR). In addition, in some of the literature, a simple CCD camera is used to detect the magnetic particles if they are detectable by such means (Morozov et al. 2007; Morozova and Morozov 2008; Mulvaney et al. 2009; Shlyapnikov et al. 2010). For many of these "magnetic label" detection methods the ability to manipulate the magnetic particles is vital, as oftentimes the detection of particles must be performed on a substrate, and the ability to quickly and easily bring magnetic particles to a desired substrate with an external magnet is a major advantage. Table 2 lists numerous examples of the different techniques used for magnetic particle detection, the kinds of magnetic particles used, the biological targets, and the sensitivity reported in the literature today.

\section{Giant Magnetoresistance Detection}

Giant magnetoresistance (GMR) is the first of the Anisotropic Magnetic Resistance (AMR) phenomena we will look at. GMR is one of the more common methods of detecting magnetic particle labels in biosensors. Essentially, the observed phenomenon of GMR is a measurable change in the resistance of a material when a small magnetic field is applied. For giant magneto resistive sensors the change in resistance due to a fairly small number of particles is detectable, and the effect increases with the number of magnetic particles resting on the sensor. The idea for the setup of a GMR biosensor is to attach a binding ligand to the substrate directly above the GMR sensors, and another binding ligand to the magnetic particles. When the target is present, it binds the magnetic particles to the substrate in an amount proportional to the amount of target. Finally, the presence of those magnetic particles results in a detectable change in the resistance of the GMR sensor, a change proportional to the amount particles attached to the surface. Figure 6 shows fabricated GMR sensors and the essential process for how the GMR phenomenon can be used to detect the presence of biological agents. 
In 1998 , an idea for an

integrated system of biosensors relying on this method of detection was published (Baselt et al. 1998). The name they gave to their system was the Bead Array Counter (BARC). The setup for the BARC used a 64 sensor array of $80 \times 5 \mu \mathrm{m}$ GMR sensors for the detection of magnetic beads. Each of the GMR
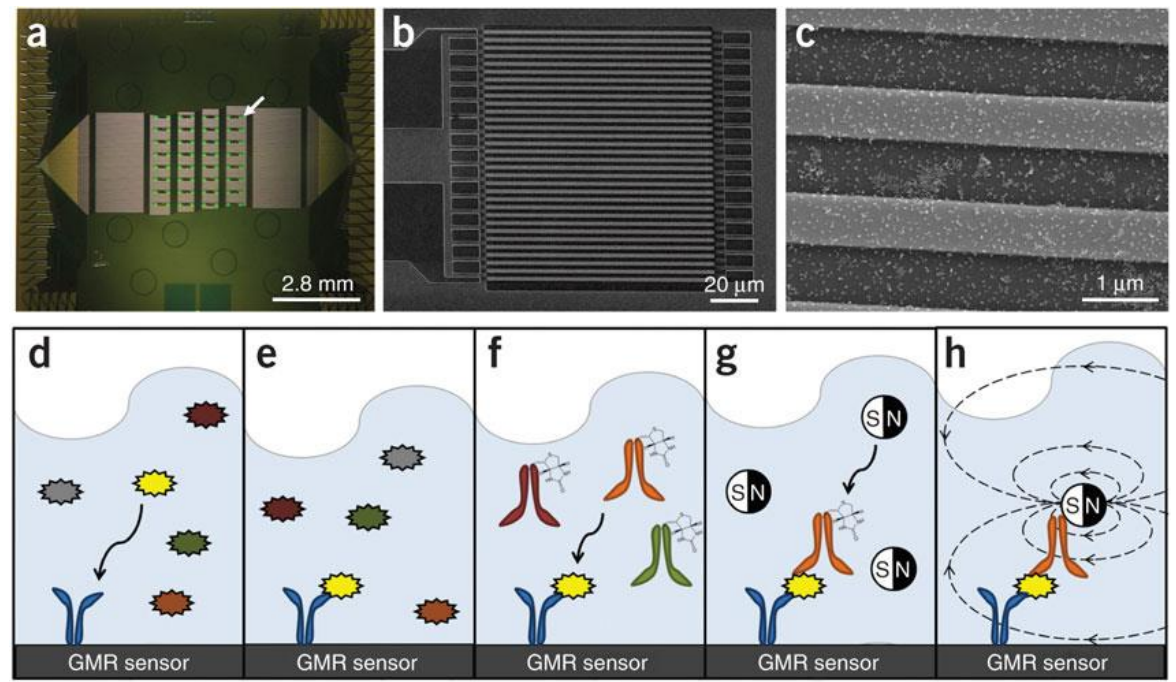

Figure 6: GMR sensors are small fabricated $(a-c)$ devices that change their resistance under the influence of magnetic fields. The use of GMR as a biosensor $(\mathrm{d}-\mathrm{h})$ involves using a biological target to attach magnetic particles to the surface such that their presence can be detected by the GMR sensor.

sensors was incorporated into a Wheatstone bridge circuit and amplified for the detection of the smallest voltage variations. The initial sensitivity of the system was around 100 particles. In order to remove beads nonspecifically bound to the surface, the device was capable of deploying a magnetic field that created a vertical force on the beads and removed the vast majority of beads that had non-specifically stuck to the surface, but left the beads bound by the target attached to the sensor surface. One of the great advantages of using the BARC setup is the great capacity for parallelization, as a large array of GMR sensors might be able to detect $\sim 1000$ analytes for a given sample. The BARC concept went through many iterations and development after the original paper was published (Miller et al. 2001; Rife 2003). In recent years, the BARC setup has been successful in detecting a great range of proteins and nucleic acids, and demonstrated the multiplexing capacity envisioned by its creators (Gaster et al. 2009; Mulvaney et al. 2007; Osterfeld et al. 2008; Schotter 2004).

Other iterations of the BARC design have used the fluid force discrimination (FFD) technique to remove nonspecifically bound particles from the sensor surface (Baselt et al. 1998; Edelstein et al. 2000). Here, the idea is that the sensor surfaces are used to capture the target and then are placed in a fluid cell undergoing laminar flow and the magnetic beads functionalized to bind the target are washed over the sensor substrate, often with an external magnet ensuring that the particles stay in close proximity to the surface. If the magnetic beads encounter the target bound to the substrate, they will quickly bind to the 
surface; otherwise they will simply be ushered along by the flow of the cell. Over a small amount of time, the sensors with capture targets will capture far more magnetic beads that

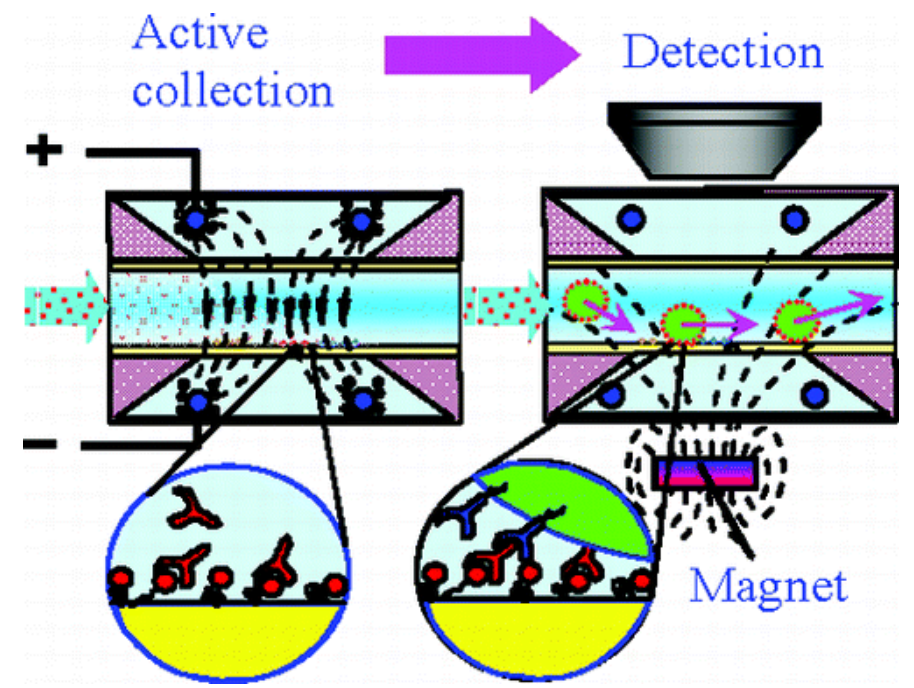

Figure 7: Fluid Force Discrimination in conjunction with optical detection methods uses similar principles as the BARC design, but does away with the magnetoresistive elements. Though the magnetic fields of the particles are not detected, the ability to force them to surface is vital to optimal function of this biosensor. can, in turn, be detected by the GMR sensor. FFD assays have also detected the magnetic beads by using a simple CCD camera (Morozova and Morozov 2008; Mulvaney et al. 2009). Figure 7 shows a setup from (Morozov et al. 2007), which outlines a magnetic particle biosensor using FFD and detection with a CCD. The use of a CCD makes for simpler experimental detection, but doesn't possess the scope for parallelization of the GMR sensors.

A limitation of GMR sensors is the essential give-and-take between the different dimensions of the biosensor. In order to make an effective biosensor, the area of the sensor substrate should be as large as possible, in order to interact and bind with a greater number of targets. However, sensitivity of the sensor increases as the magnetoresistive strip becomes thinner, with a smaller surface area (Baselt et al. 1998). This means that a balance must be struck between the area available for analyte detection and the sensitivity of the GMR sensor itself.

\section{Single Particle Detection Methods}

As GMR sensors have gained some recognition in biosensor platforms, several new techniques capable of detecting single particles have arisen, all of which rely on some kind of anisotropic magnetic resistance (AMR) phenomenon. The motivation behind such techniques is that they offer the capacity for extremely sensitive target detection. However, many of these techniques are only just beginning to advance into actual detection of biological targets, because of the small detection area associated with such sensors.

The first of the single particle detection technologies is the sub-micron GMR sensor (Wood et al. 2005). The essential mechanism is the same as for larger GMR sensors but the dimensions are drastically reduced to around $2 \mu \mathrm{m} \times 100 \mathrm{~nm}$. The reduced size of the 
sensors makes it possible for the magnetic field generated from a single $100 \mathrm{~nm}$ magnetic particle to cause a detectable voltage difference across the GMR sensor.

Spin valves are another method of single particle detection that has been widely investigated (Ferreira et al. 2003; Heim et al. 1994; Li et al. 2003; Li et al. 2006; Loureiro et al. 2009). Spin valves rely on an anisotropic magnetic resistance (AMR) in order to detect very small magnetic fields. The essence of spin valve technology is a coupling of ferromagnetic thin films across a non-magnetic metal layer (Dieny et al. 1991; Ferreira et al. 2003; Speriosu et al. 1991). In spin valves, the resistance of the material changes depends on the angle between the magnetization directions of the two ferromagnetic layers. Most spin valves today also use an additional ferrimagnetic layer to "pin" the orientation of the magnetic moment of one of the ferromagnetic layers. Figure 8 shows the basic setup of the sensor. The single

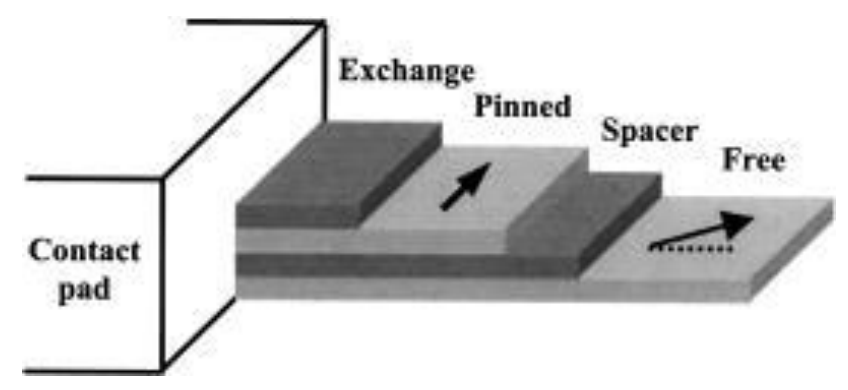

Figure 8: A spin valve consists of a "free" ferromagnetic layer, a non-ferromagnetic metal "spacer" layer, another "pinned" ferromagnetic layer, and a ferrimagnetic "exchange" layer that pins the previous ferromagnetic layer

magnetic particle can then be detected by the way it affects the orientation of the magnetic moment of the other ferromagnetic layer. Of all the single particle detection mechanisms, spin valves are among the most researched and furthest along in detection of biological agents. Spin valves have been both employed in microarrays of sensors (Wang et al. 2005) and used to detect the presence of DNA oligonucleotides (Loureiro et al. 2009).

The Magnetic Tunnel Junction (MTJ) is another type of single particle sensor with a design similar to that of spin valves (Grancharov et al. 2005; Shen et al. 2005). The difference in structure between the two types of sensor is that the non-ferromagnetic layer of the spin valve is replaced with a "tunnel barrier" in the MTJ (Brzeska et al. 2004; Moodera et al. 1995). In these sensors the current cannot conduct across the intermediate layer, the electrons must effectively tunnel through the barrier. Similar to spin valves, the amount of current that gets through the intermediate layer is dependent on the relative orientations of the magnetizations on either side of the tunnel barrier. The presence of magnetic particles changes the tunneling efficiency and consequently the conduction current through the sensor. Like the related spin valves, MTJs have also been employed in microarrays (Wang et al. 2005) and demonstrated success in detecting DNA oligonucleotides (Shen et al. 2008). 


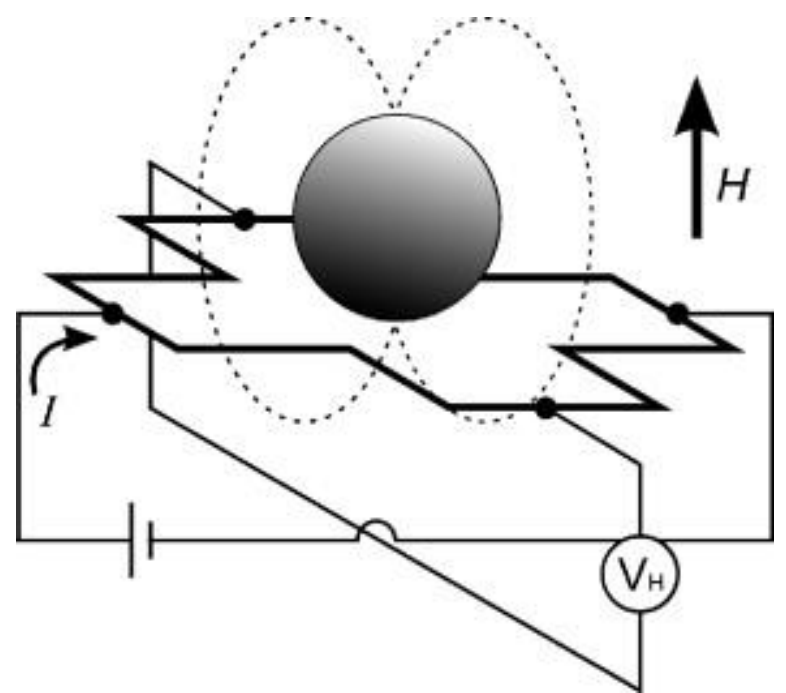

Figure 9: Sensors based upon the Hall Effect take advantage of the magnetic force exerted on charge carriers in flowing current. This magnetic force causes a detectable transverse voltage to be formed across the Hall sensor. An external magnetic field is usually required in order to magnetize the particle sufficiently that its magnetic field can be detected.

to the direction of current flow. The field from a single magnetic particle is sufficient to generate a transverse voltage across specially designed Hall probes. Often, Hall sensors are used in conjunction with an applied external field in order to magnetize the particles sufficiently that they can be detected. Figure 9 shows an example of how magnetic particle can be magnetized by a DC field in the vertical direction. Arrays of Hall probe sensors have shown effectiveness in detecting some biological targets (Aytur et al. 2006).

The final technique of single particle detection that employs an AMR effect is the AMR ring sensor. Essentially, these sensors are an AMR material deposited in a ring pattern such that a single particle could fit within the ring's inner radius (Jiang et al. 2006; Miller et al. 2002). Ring sensors take advantage of the axial symmetry of the fields of magnetic particles. The rings are constructed
The Hall Effect has also been shown to have the capacity to detect the magnetic fields from single particles as well (Besse et al. 2002; Ejsing et al. 2004; Mihajlović et al. 2005). The Hall Effect, in essence is the generation of a transverse voltage across a conducting material in the presence of magnetic field. The Hall Effect results from the magnetic force acting upon moving charge carriers in a direction perpendicular to the direction of

current flow. The magnetic force on the charge carriers causes them to tend to "stack" on one side of the conductor as they flow. The uneven distribution of charge carriers in the conductor results in a measureable voltage perpendicular

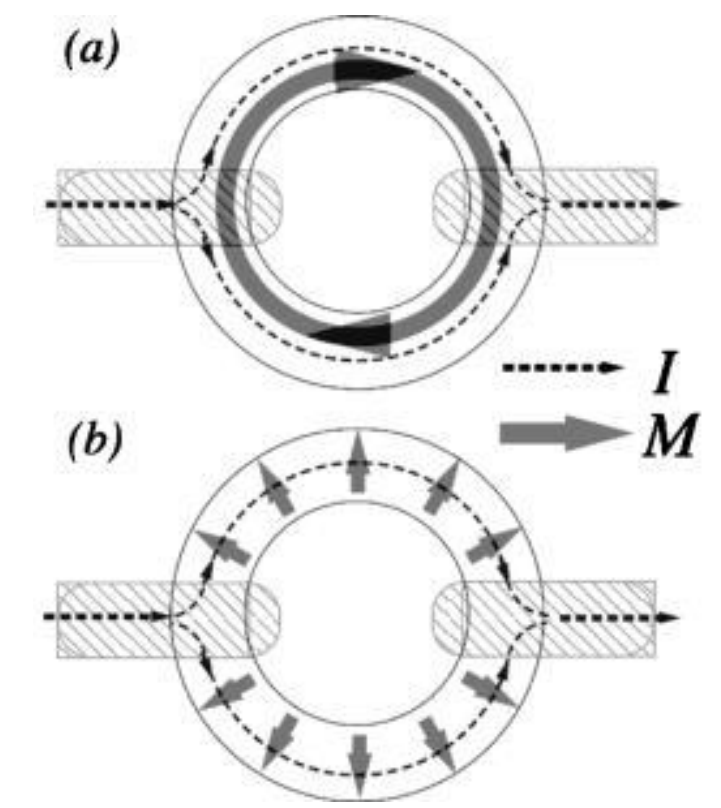

Figure 10: Ring AMR sensors are made from magnetoresistive material deposited in a ring shape. The geometry of the ring sensor makes it more sensitive to the magnetic fields generated by single particles. Part (a) shows the magnetization of the ring when no particles are present (high resistance), and (b) shows the magnetization when a particle rests in the middle of the sensor (low resistance). 
such that when no particle is present, the ring sensor is in a high resistance state, because the magnetization is parallel to the direction of current flow. When a magnetic particle is present, the radial field of the particle modulates the magnetization such that it becomes perpendicular to the direction of current flow, putting the sensor into a low resistance state.

\section{Magnetic Coil Detection}

Magnetic coils set up in certain kinds of circuits have successfully been used to detect the presence of magnetic particles bound to a substrate. Magnetic particles have a different magnetic permeability than that of air and hence change the inductance of a solenoid coil when they are placed within the interior of the coil (Kriz, Radevik, and Kriz 1996). The setup

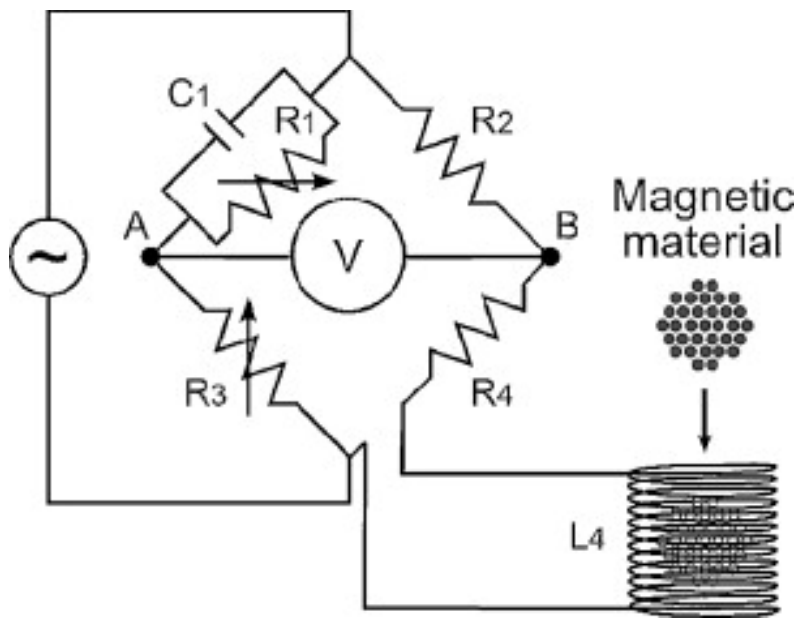

Figure 11: One setup of using inductance coils to detect magnetic particle sensors uses a Maxwell Bridge. In this circuit, a change in the inductance of the coil caused by the addition of the magnetic particles results in a voltage difference between points $A$ and $B$.

for this method of detection involves

placing target ligands on a simple substrate-generally plastic or glass, but silica microparticles have also been used (Kriz, Gehrke, and Kriz 1998)-and binding the magnetic

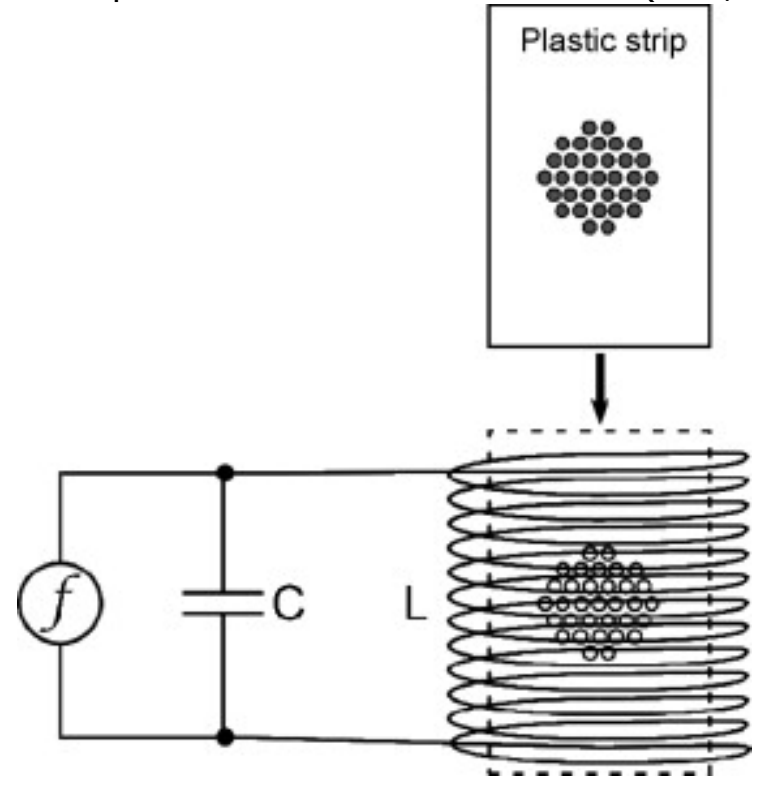

Figure 12: An alternative method to detecting inductance change in a magnetic coil is with resonant $\mathrm{LC}$ circuit. $\mathrm{A}$ change is observed in the resonance frequency of the circuit upon the addition of the magnetic particles. particles to the substrate with the target. The substrates can then be placed into the coil and the number of magnetic particles can be detected by observing the changes in the inductance of the solenoid coil.

There are multiple ways of detecting the changes in the inductance of the detection coil. The first is through the use of a Maxwell Bridge circuit (Kriz, Radevik, and Kriz 1996; Kriz, Gehrke, and Kriz 1998). Essentially, this setup is an RLC circuit with several variable resistances, being driven with an alternating current source. The resistors can be adjusted such that the output of the circuit is zero before the sample is inserted into the coil. Placing 
magnetic material into the coil results in a voltage change at the output of the circuit, a change that is proportional to the amount of magnetic material placed in the interior of the coil. Figure 11 shows the basic circuit schematic used in this method of detection

The other method of detecting the change of inductance in a solenoid coil is by setting up an LC resonant circuit (Richardson et al. 2001). In this case, the change in the inductance of the coil, resulting from the presence of the magnetic particles, corresponds to a change in the resonant frequency of the circuit. The change of the circuit's resonance frequency can be detected and corresponds to the number of magnetic particles in the sample. Figure 12 shows the circuit schematic for this detection mechanism.

These detection methods have been used to detect many biologically relevant molecules like C-reactive protein (Kriz et al. 2005), Human Transferrin (Richardson, Hawkins, and Luxton 2001), and Toponin I (Kiely et al. 2007). This technique is limited by the large number of magnetic particles required for detection (compared to other detection techniques). Initial investigations reported that at least $10^{5}$ magnetic particles are required for detection(Richardson et al. 2001).

\section{Cantilever Detection}

Micro-cantilevers as biosensors have been widely researched in the past several years. Cantilevers have been used to detect the presence of single cells, and even single cell growth (Boisen and Thundat 2009; Fritz 2008; Ziegler 2004). Recent work has used magnetic particles in conjunction with cantilever technology as a biosensor (Weizmann et al. 2005; Weizmann et al. 2004). The apparatus for this detection method consists of using the target to bind magnetic particles to the surface of a cantilever. An external magnet is placed near the cantilever and the deflection of the cantilever can be measured by bouncing a laser beam of the surface of the cantilever and measuring its displacement on the photodiode array. The cantilever will be deflected in proportion to the number of magnetic particles attached to the surface, and the number of magnetic particles is
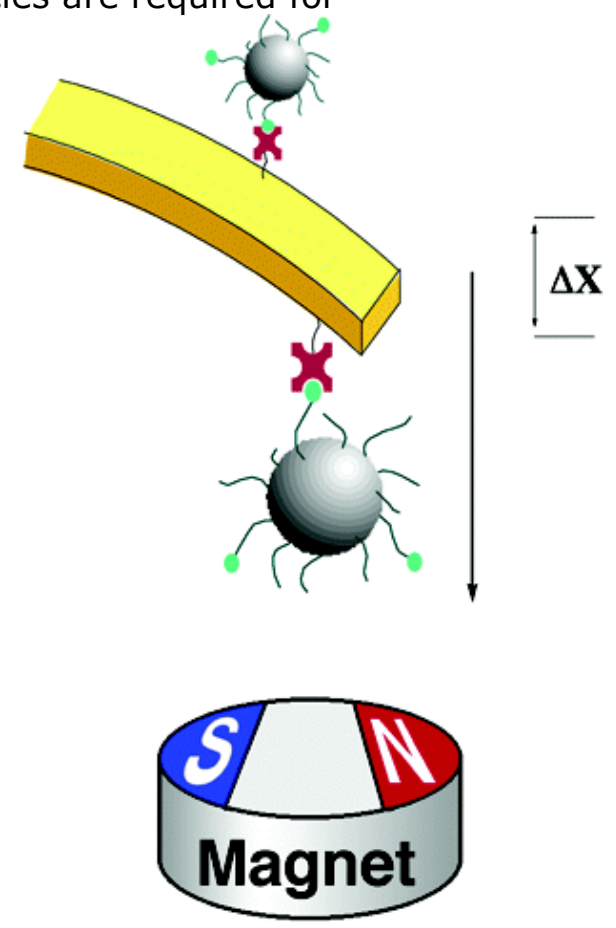

Figure 13: Magnetic particles can be used to detect biological targets in conjunction with cantilevers by binding the magnetic particles to the cantilever with the target and measuring the cantilever deflection when an external magnetic field acts on the magnetic particles attached to the cantilever. 
proportional to the amount of target present in the incubation. Figure 13 shows the basic setup by which cantilever detection functions.

\section{SQUID Detection}

Superconducting Quantum Interference Devices (SQUIDs) are used to measure the magnetic relaxation of materials. In other words, they measure the time it takes for a given material to lose its magnetization after being saturated by an external magnetic field, or if it does not lose its magnetization, what is the remanent magnetization of the sample. For

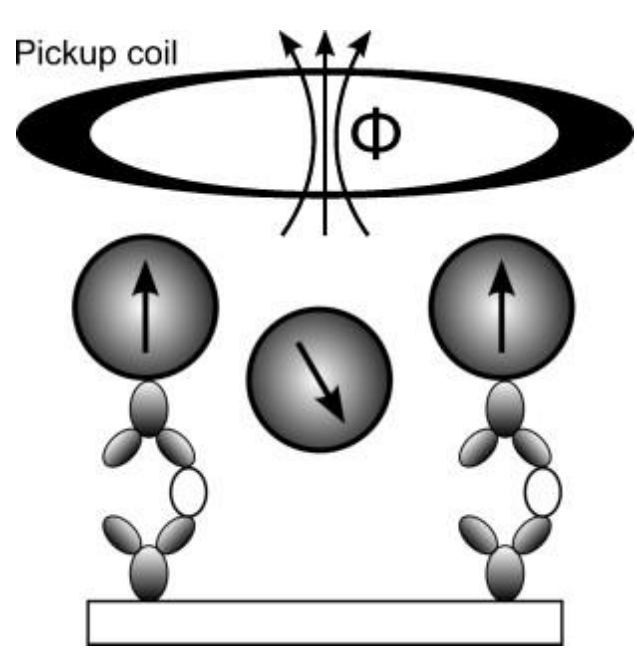

Figure 14: Detection of bound magnetic particles with SQUID exploits the fact that bound particles are not able to disperse themselves through diffusion and their remnant magnetic relaxation will be much slower than for unbound particles. situations involving magnetic particles diffusing through a solution, the magnetization of the system will eventually return to zero. Magnetic remanent relaxation occurs in one of two forms: Brownian and Néel relaxation (Fannin et al. 2006). Brownian relaxation is the rearrangement of the magnetic moments that occurs due to the diffusion of the particles. Since the particles are free to diffuse, their magnetic moments will randomly reorient themselves as the particles diffuse. Néel relaxation occurs when the particles (or material) are not free to diffuse and hence the internal magnetization of the material must realign itself due to ambient thermal energy. Néel relaxation is generally slower than Brownian relaxation for the systems of interest.

Detection of relaxation times can detect the presence of biological targets in different ways. The first method of detection involves using the biological target to bind the magnetic particles to a substrate (Kotitz et al. 1997; Tsukamoto et al. 2005). In this case the particles that are bound will have to relax via the Néel mechanism, while unbound particles can relax using the much faster Brownian mechanism. By detecting the extent of Néel relaxation against Brownian, SQUID measurements can detect the amount of target analyte present. The basic scheme for this detection process can be seen in Figure 14. 
Another method of detecting the presence of biological targets with SQUID is by using the binding analytes to form clusters of particles (Strömberg, Göransson et al. 2008; Strömberg, Zardán Gómez de la Torre et al. 2008). While these clusters will still be free to reorient due to diffusion, they will do so more slowly than individual, non clustered particles. The detectable change in relaxation time can be analyzed in such a way that it corresponds to differences in the amount biological target. SQUID techniques have been successful in detecting both proteins (Chemla et al. 2000; Enpuku et al. 2005; Enpuku et al. 2007; Kotitz et al. 1997; Kuma et al. 2010) and oligonucleotides (Strömberg et al. 2009; Zardán Gómez de la Torre et al. 2010). The substrate free version of SQUID detection has relied on a DNA amplification technique, "Rolling Circle Amplification (Gusev et al. 2001)" in order to form the clusters of magnetic particles needed for this method of detection. Figure 15 shows how RCA can be used to detect DNA in conjunction with SQUID.

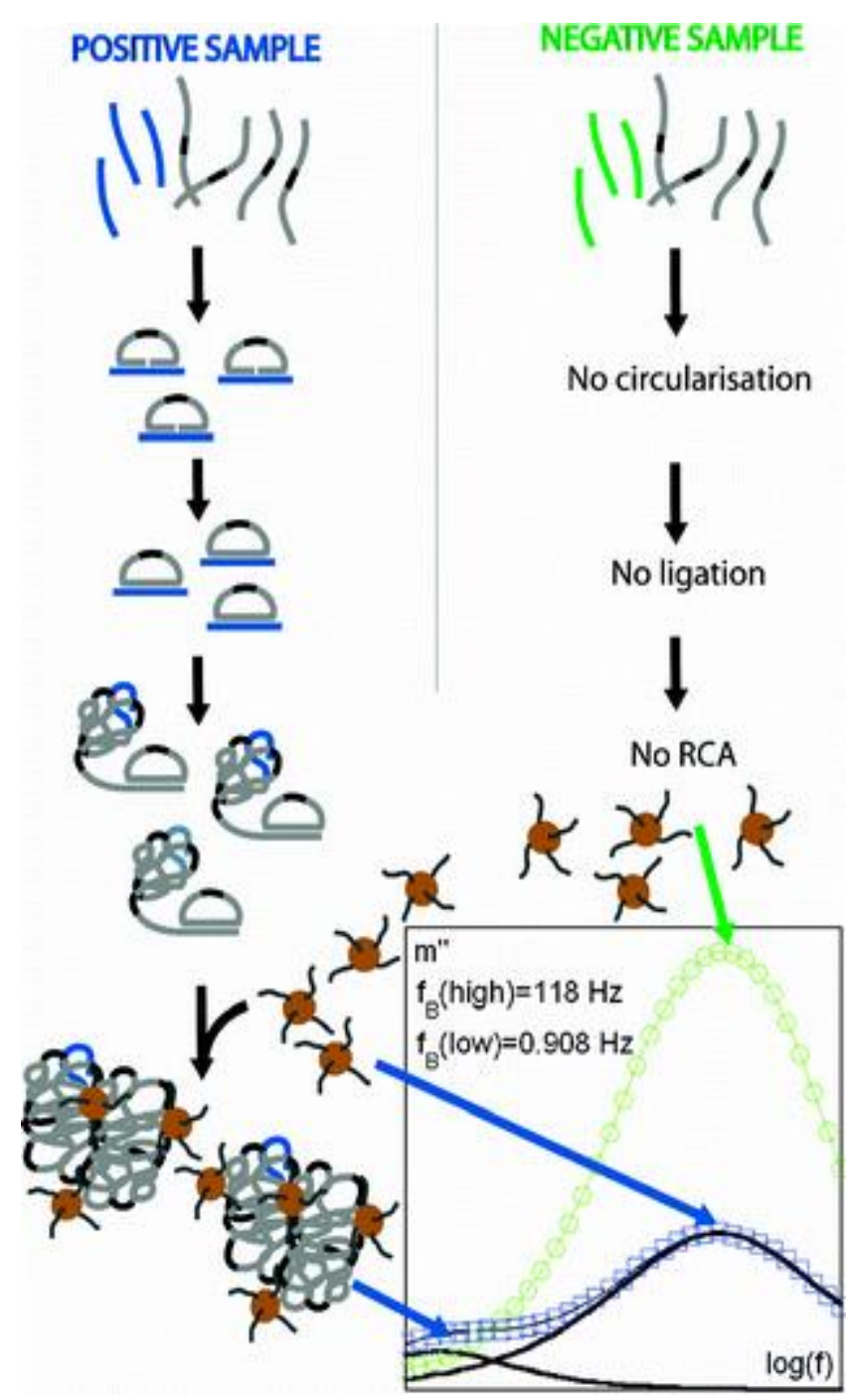

Figure 15: Detection of DNA using SQUID has been demonstrated without the use of a substrate to bind the particles. For this detection method, the target DNA is amplified into large coils that then bind the particles together in clumps resulting in a change in the Brownian relaxation frequency.

\section{Spin Relaxation Detection with NMR}

Nuclear Magnetic Resonance (NMR) is another technique that has been used to detect the presence of biological targets bound to magnetic particles without a substrate (Grimm et al. 2004; Perez et al. 2002). In essence, the setup is similar to the substrate-free SQUID detection mechanism: targets bind the magnetic particles together into clusters and the presence of clusters can be measured by the apparatus. For NMR detection though, the system takes advantage of the fact that the spin-spin relaxation times of the protons in the water is reduced by larger clusters of magnetic particles. Thus, when the binding target is 


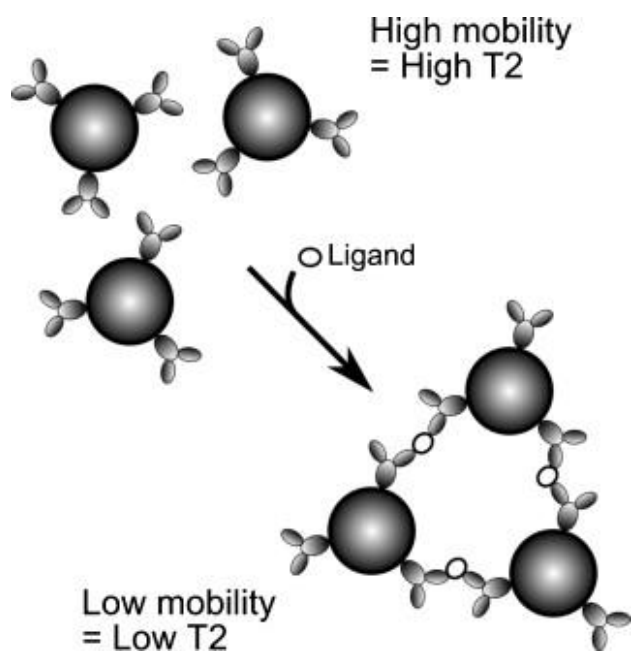

Figure 16: Magnetic particles clump together in the presence of the biological target. This clumping effect causes a change in the spin-spin relaxation times to change in a detectable fashion present, the spin-spin relaxation will be lower than for samples without the binding analyte. Figure 16 gives a basic schematic for how biological targets can be detected with the spin relaxation method. The differences in spin relaxation can be easily detected with simple benchtop relaxometer devices (Perez et al. 2002). It is also possible that this method of detection could be performed in vivo using larger MRI machines.

Many biological systems have been investigated using spin relaxation. DNA oligonucleotides (Perez et al. 2002), Proteins (Grimm et al. 2004), whole cells (Kaittanis, Santra, and Perez 2009), and cellular growth (Kaittanis, Nath, and Perez 2008) have all been detected using the spin relaxation NMR method.

\section{Conclusion}

Magnetic particles have been used as key components in a vast number of different biological sensing applications. That so many different methods have been devised in a relatively short period of time gives an indication of their versatility and usefulness in conjunction with biological systems. Up to this point, the vast majority of magnetic particle biosensors have measured the presence of biological targets such as DNA, proteins, or entire cells. Next we will turn to a detailed look at a technique that has shown great promise in applications involving the measurement and growth of individual cells.

\section{Asynchronous magnetic bead rotation (AMBR) biosensors and biomedical applications}

\section{Introduction}

A rapid, high throughput cell-growth/cell-stress biosensor with high resolution and small sample volumes could serve a wide area of biomedical research and applications. Such biosensors would be valuable for heterogeneity studies of clonal populations, drug screening, clinical antimicrobial susceptibility testing (AST) of bacteria, and for determining optimal cancer treatments. Certainly, new automated instruments for rapid and accurate recognition of bacterial resistance to antibiotics are needed, as was pointed out in the review of contemporary AST practices (Jorgensen and Ferraro 2009); while rapid molecular diagnostic methods exist, they have limited utility and there is a need for rapid phenotypic 
measures of antimicrobial susceptibility (Jorgensen and Ferraro 1998, 2009). The AMBR biosensor offers rapid phenotypic results, namely the minimum inhibitory concentration (MIC), which can be used to determine the exact amounts of antibiotics needed on the patient to patient level. On the other hand, while chemotherapy sensitivity and resistance assays (CSRAs) for cancer are not yet widely used in clinical decision making (Schrag et al. 2004), they have a significant potential to be used for determining the right therapy on an individual patient basis; however novel rapid growth/stress sensors are needed. While technologies with resolution of single cell growth do exist, such as bright field microscopy, AFM, SEM and cantilevers, scalability and automation remain a major hurdle. AMBR can be seamlessly combined with immunomagnetic separation, since the same magnetic particles can be used for both. AMBR biosensors show a great potential for various kinds of microand nano-growth studies, owing to their scalability, simplicity and nanometer scale resolution. The following sections discuss AMBR sensor applications from the Kopelman laboratory in more detail.

\section{Applications of magnetic particles in rotating magnetic fields}

Magnetic particles driven with rotating magnetic fields have interesting qualities that have found several applications in biomedical engineering. The applications include more traditional ones, such as magnetic microdrills (Honda et al. 1996; McNaughton, Anker, and Kopelman 2005), micromixing (Biswal and Gast 2004), and artificial bacterial flagella (Ghosh and Fischer 2009; Zhang et al. 2009), as well as less straightforward applications, such as magnetic separation (Yellen et al. 2007), background extraction (Anker, Behrend, and Kopelman 2003), biomolecule detection (Hecht et al. 2011; Park, Handa, and Sandhu 2010), viscosity measurement (Gaub and McConnell 1986), and asynchronous magnetic bead rotation (AMBR) biosensors, which are based on the rotational frequency of magnetic particles in rotating magnetic fields (Elbez et al. 2010; Kinnunen et al. 2010; McNaughton, Agayan, Clarke et al. 2007; McNaughton et al. 2006; McNaughton et al. 2009).

Another field of research worth mentioning in this context, and which precedes all the previously mentioned studies, is ferrohydrodynamics, where magnetic nanoparticle ensembles in rotating magnetic fields have been extensively studied. The first such study was conducted by Moskowitz and Rosensweig in 1967, where then recently discovered ferrofluids were placed in a rotating magnetic field and their rotation rates were observed (Moskowitz and Rosensweig 1967). It was soon followed by a theoretical study on single ferromagnetic (FM) and superparamagnetic (SPM) particles in rotating magnetic fields, so as to answer some of the questions that were raised (Caroli and Pincus 1969). Caroli and Pincus theoretically predicted that there exists a critical frequency for FM particles, only below which the particles rotate synchronously with the rotating field, anticipating recent work (McNaughton et al. 2006). Two decades later Popplewell et al., using numerical integrations of the equations of motion, found that above the critical driving frequency the FM particle rotation rate is steady, but with a superimposed oscillatory component of relatively small magnitude; furthermore, the mean particle rotation rate decreases as the driving frequency increases (Popplewell, Rosensweig, and Johnston 1990). This anomalous rotational phenomenon, where the magnetic particle rotates asynchronously with the driving field, is what the asynchronous magnetic bead rotation (AMBR) biosensors are based on, 
and which will be discussed in more detail in the following sections. Applications of synchronous and asynchronous magnetic bead rotation are shown in Figure 17.

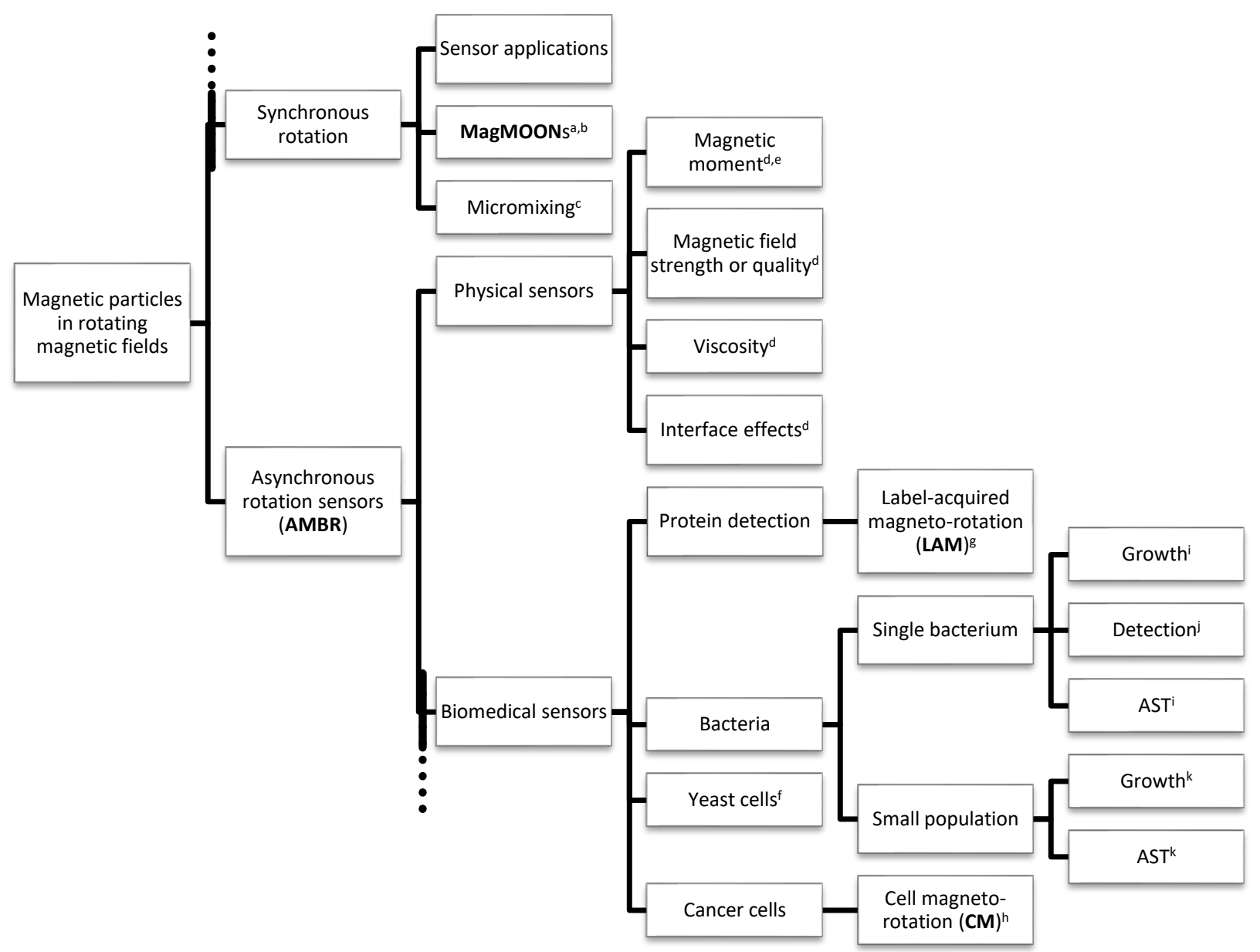

Figure 17: AMBR biosensor- and other applications of magnetic particles in rotating magnetic fields.

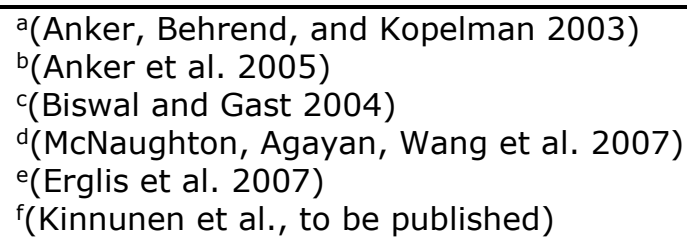

\section{AMBR biosensors}

\section{Theory}

A ferromagnetic particle has a tendency to align with an external magnetic field, and therefore, at low driving frequencies, the magnetic particle rotates in synchrony with a rotating magnetic field. Above some threshold (called the critical frequency), viscous forces of the surrounding fluid hinder the synchronous rotation sufficiently, forcing the particle to rotate briefly backwards every time the driving field laps around the particle. In the case of two preferred orientations in the driving field (elongated superparamagnetic particles 
(Tierno et al. 2009) and optically driven nanorods (Shelton, Bonin, and Walker 2005) the backward rotation happens twice every time the particle is lapped by the field. An example of a ferromagnetic particle rotating synchronously (below the critical driving frequency) and asynchronously (above the critical driving frequency) with the external rotating magnetic field can be seen in Figure 18 (McNaughton et al. 2006).

(a)

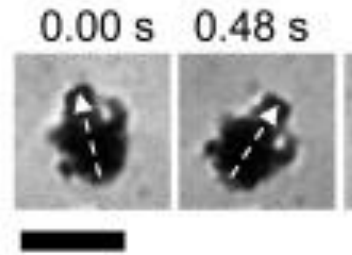

(b)

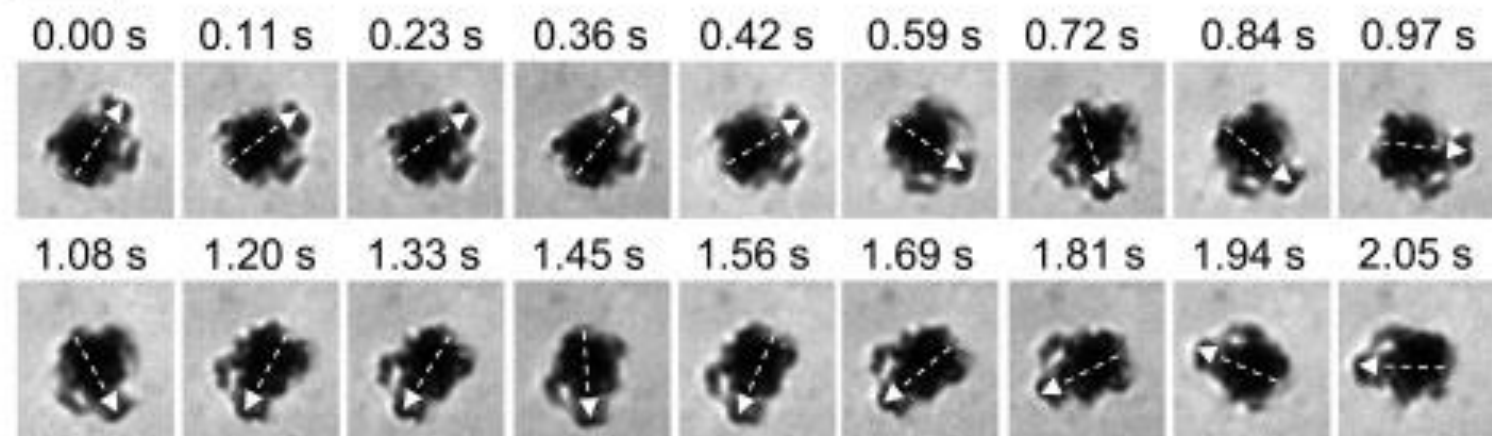

Figure 18: Sequence of bright-field microscopy images of a single magnetic particle, where the black scale bar is 7 um. (a) The particle is driven at below the critical driving frequency, rotating in the clockwise direction. (b) The particle is driven above the critical driving frequency. The particle begins in the clockwise direction, $t=0.00-0.23 \mathrm{~s}$ but then rotates in a direction that is opposite of the external driving field, the counterclockwise direction, at $\mathrm{t}=0.36,0.84,0.97,1.45$, and $2.05 \mathrm{~s}$.

\section{Ferromagnetic AMBR}

To derive the critical frequency one can start from the equation of motion, which for a FM particle in a viscous solution in an applied magnetic field is

$I \frac{d^{2} \theta_{F M}}{d t^{2}}+\gamma \frac{d \theta_{F M}}{d t}=m B \sin (\phi)$

where $I$ is the moment of inertia, $\theta_{F M}$ is the magnetic moment, $t$ is time, $\gamma$ is the drag coefficient, $m$ is the permanent magnetic moment of the particle, $B$ is the magnetic field strength and $\phi$ is the phase lag, which is the angle between the external magnetic field and the magnetic moment (Valberg and Butler 1987). See Figure 3 for a schematic representation of the system.

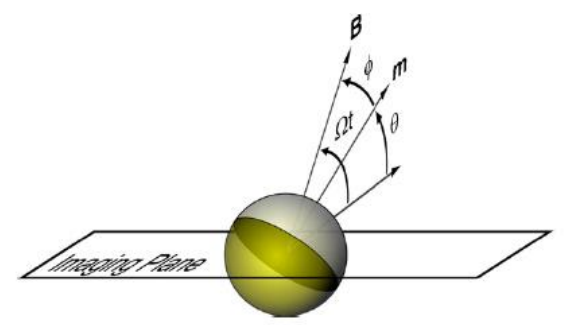

Figure 19: Schematic representation of a rotationally driven magnetic particle.

In a rotating magnetic field, the magnetic torque acting on the particle is $\Gamma_{m}=|\mathbf{m} \times \mathbf{B}|=m B \sin (\phi)=m B \sin \left(\Omega t-\theta_{F M}\right)$, where $\Omega$ is the frequency of the rotating magnetic field. In low Reynolds number environments the inertia term can be ignored and 
equation (1) can be written in a nondimensional form, which is the nonuniform oscillator equation:

$\frac{d \phi}{d \tau}=\frac{\Omega}{\Omega_{c}}-\sin (\phi)$,

where $\Omega_{c}=m B / \gamma, \tau=\Omega_{c} t$, and $\phi=\Omega t-\theta_{F M}$. Below the critical driving frequency $\Omega_{c}$, the particle rotates in synchrony with the driving field with a phase lag $\phi=\sin ^{-1}(\gamma \Omega / m B)$. The maximum phase lag $\pi / 2$ occurs when $\Omega=\Omega_{c}$.

In the above mentioned systems the critical frequency and the decline in the time averaged particle frequency, with increasing driving frequencies, has a well characterized dependence on the driving frequency, namely:

$$
\left\langle\dot{\theta}_{F M}\right\rangle=\Omega-\sqrt{\Omega^{2}-\Omega_{c}^{2}}
$$

where $\left\langle\dot{\theta}_{F M}\right\rangle$ is the time average of the particle rotation. This relationship has been reported for magnetic particles in rotating magnetic fields, namely for magnetic hole systems (Helgesen, Pieranski, and Skjeltorp 1990), magnetorheological fluid droplets (Bacri, Cēbers, and Perzynski 1994; Lācis et al. 1997), magnetically loaded carbon nanotubes (Korneva et al. 2005), ferromagnetic particles (McNaughton et al. 2006), and elongated paramagnetic particles (Tierno et al. 2009). Nonmagnetic systems such as optically driven nanorods have also been reported to have similar frequency behavior (Shelton, Bonin, and Walker 2005).

While the dynamics of magnetic particles in rotating magnetic fields had been studied previously quite extensively, the first to recognize the potential of asynchronously rotating magnetic beads for biosensing applications were McNaughton et al (McNaughton et al. 2006). The biosensing ability of the ferromagnetic AMBR biosensors stems from the fact that the critical frequency, and therefore the rotational frequency in the asynchronous regime, is a function of the fluidic drag coefficient $(\gamma=\kappa \eta V)$, and therefore the volume:

$$
\Omega_{c}=\frac{m B}{\kappa \eta V},
$$


where $\kappa$ is the shape factor (which is 6 for a sphere), $\eta$ is the dynamic viscosity, and $V$ is the total volume of the rotating body. As a result, a ferromagnetic particle in a rotating magnetic field with a frequency above the critical driving frequency has a well-defined rotation rate, which depends on its effective volume $\left(V_{\text {eff }}=\kappa V\right)$.

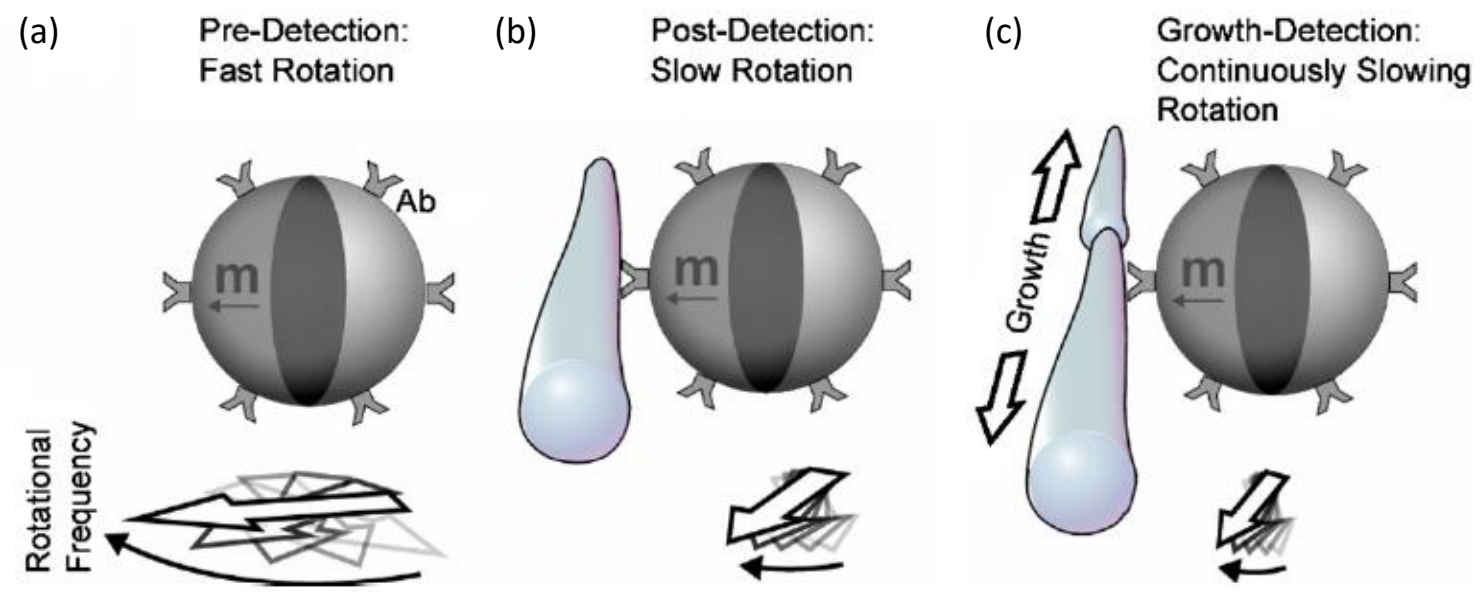

Figure 20: Schematic representation of a magnetic microsphere functionalized with an antibody with (a) no attached bacteria, (b) with one bacterium and (c) after bacterial growth. The rotation rate slows as more bacteria are attached or grow on the surface of the particle.

The effective volume can be solved using equations 3 and 4:

$V_{e f f}=\frac{m B}{\eta\left\langle\dot{\theta}_{F M}\right\rangle \sqrt{2 \Omega /\left\langle\dot{\theta}_{F M}\right\rangle-1}}$.

Therefore, in controlled environments, where the temperature, magnetic field amplitude and frequency are kept constant, the effective volume can be accurately measured by simply observing the rotation rate.

Similarly, the AMBR sensor can be used to measure other variables in equation 5 , such as the viscosity, magnetic moment and magnetic field strength. It is also possible to use the phase lag of a synchronously rotating magnetic particle as a biosensor (Romano et al. 2003). However, measuring the oscillatory phase of a physical system is in general more difficult and less precise than measuring frequency.

\section{Superparamagnetic AMBR}

Magnetic particles that consist of embedded superparamagnetic (SPM) nanoparticles, with an induced rather than permanent magnetic moment, are preferentially used for immunomagnetic separation. A rotating magnetic field exerts a time invariant torque on a collection of SPM nanoparticles (Janssen et al. 2009), and therefore these magnetic particles also lend themselves for AMBR biosensor applications. For instance, depending on the size distribution of its embedded magnetic nanoparticles, a given magnetic microparticle can exhibit ferromagnetic or SPM behavior in a rotating magnetic field. Ferromagnetic behavior 
will be apparent in the low amplitude and low frequency regime, while an SPM torque behavior will be dominant in a field with high amplitude or frequency. The total magnetic torque exerted on a particle in a rotating magnetic field can be written as:

$$
\begin{aligned}
\boldsymbol{\tau}_{m a g} & =\mathbf{m}_{p e r m} \times \mathbf{B}+\left[\left(\chi^{\prime}+i \chi^{\prime \prime}\right) V_{m} \mathbf{B}\right] \times \mathbf{B} \\
& =m_{p e r m} B \sin \left(\Omega t-\theta_{F M}\right) \hat{\mathbf{e}}+\chi^{\prime \prime} V_{m} \frac{B^{2}}{\mu_{0}} \hat{\mathbf{e}}
\end{aligned}
$$

where $\chi^{\prime}$ and $\chi^{\prime \prime}$ are the real and imaginary parts of the magnetic susceptibility, $V_{m}$ is the magnetic content volume, $\mu_{0}$ is the permeability of free space, and $\hat{\mathbf{e}}$ is a unit vector of the magnetic field. The real part of the susceptibility does not contribute to the torque, because it is parallel to the applied magnetic field, and the cross product is therefore zero at all times. The imaginary susceptibility however is perpendicular to the rotating magnetic field, and the cross product is therefore nonzero. The imaginary susceptibility is a property of the magnetic particle, and has a frequency dependency that is depicted by the embedded nanoparticle size distribution. In the high frequency case the contribution from the permanent magnetic moment can be negligible and the first term on the right-hand-side of equation 6 is zero. Equating the SPM torque with the torque from the fluidic drag the rotation rate of the particle can be solved:

$$
\begin{aligned}
& \boldsymbol{\tau}_{d r a g}=-\kappa \eta V \dot{\theta} \hat{\mathbf{e}} \\
& \boldsymbol{\tau}_{d r a g}=-\boldsymbol{\tau}_{m a g} \\
& \chi^{\prime \prime} V_{m} \frac{B^{2}}{\mu_{0}}=\kappa \eta V \dot{\theta}_{S P M} \\
& \dot{\theta}_{S P M}=\frac{\chi^{\prime \prime} V_{m} B^{2}}{\kappa \eta V \mu_{0}} .
\end{aligned}
$$

Note that the backward rotations that are present in the FM asynchronous rotation do not exist in the SPM case. Also, there is no explicit driving frequency dependence in the rotation rate of the particle; however the imaginary susceptibility is a property of the magnetic material and usually is frequency dependent in nature. From equation (10) the effective volume can once again be solved for biosensing applications, yielding the effective volume of an SPM particle as a function of its rotation frequency:

$$
V_{e f f}=\frac{\chi^{\prime \prime} V_{m} B^{2}}{\eta \mu_{0} \dot{\theta}_{S P M}}
$$

The general definition of superparamagnetism is that the magnetic moments average to zero over a finite time; usually a time scale of 100 seconds is assumed (often implicitly) (Krishnan 2010). However, in a rotating magnetic field the magnetic moments cannot relax 
due to the constant magnitude of the field, and therefore this definition does not apply; in this case a natural distinction between permanent and induced magnetic moment is which of the equations, (3) or (10), fit the frequency response of the rotating particle. Equation (3) describes the response of a ferromagnetic- and equation (10) superparamagnetic particle in a rotating magnetic field.

\section{Biomedical applications of AMBR biosensors}

Using the theory described in the above sections, the AMBR biosensors have been used to measure a variety of biological subjects, and some of these experiments are discussed below.

\section{Single bacterium detection}

One of the first experiments revealing the high volumetric resolution of the AMBR biosensor was single cell detection. The binding events of individual bacteria can be measured by observing the rotation rate changes of the AMBR biosensor (McNaughton, Agayan, Clarke et al. 2007), see Figure 21. The effective volume change of the rotating body, due to the attachment of a single bacterium, resulted in 3.8 times slower rotation rate, on average.

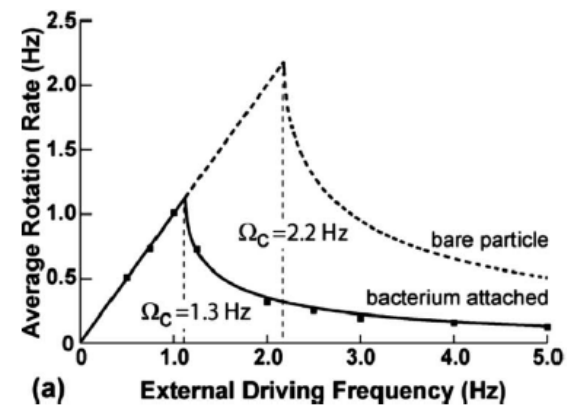

(a)

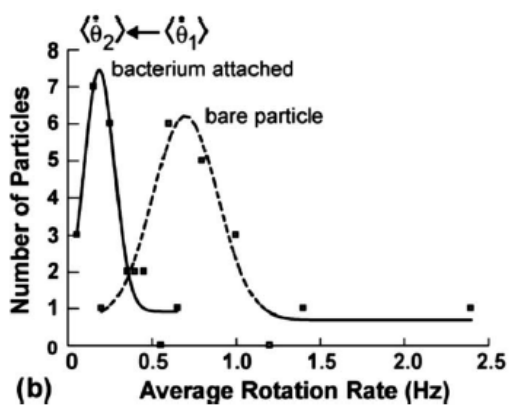

(b)

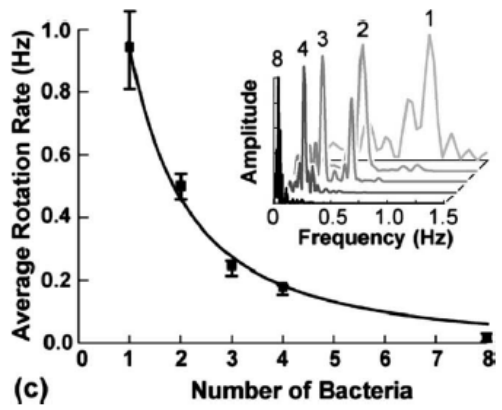

(c)

Figure 21: (a) The rotational response of a single magnetic microsphere with an attached bacterium at various external driving frequencies, where the squares are experimental data and the line is a theoretical fit to Equation 3, the dotted line is an approximated curve for a microsphere without a bacterium. (b) The average rotation frequency of 20 particles in a fluidic cell incubated with bacteria (solid curve) and a fluidic cell without bacteria (dashed curve). The magnetic microspheres with one bacterium attached rotated an average of 3.8 times slower than those without. (c) The average rotation rate of a magnetic microsphere dimer driven at $3.75 \mathrm{~Hz}$, where 1, 2, 3, 4, and 8 bacterial cells were sequentially attached. The fit corresponds to the expected change in the nonlinear frequency, determined from Equation 3 and 4 for incremental additions of volume. The inset shows the normalized power spectral density of the intensity fluctuations of the dimer with $1,2,3,4$, and 8 cells attached sequentially.

\section{Single bacterium growth (Nano-growth) and cell division}

Optical microscopy offers the ability to measure the elongation of individual bacteria over multiple generations and it is currently the most widely used tool for studying single cell behavior (Brehm-Stecher and Johnson 2004; Elfwing et al. 2004; Inoue et al. 2001). However, the spatial resolution of far-field optical microscopy techniques is limited by the diffraction of light. Other high resolution tools that have proven useful for single cell analysis include scanning probe techniques (Touhami, Jericho, and Beveridge 2004) and cantilevers 
(Bryan et al. 2010), but studies spanning multiple generations of individual cells have not yet been demonstrated with these techniques; furthermore, their optimal medium is air, not water. As an alternative approach, superparamagnetic beads have been used to monitor the growth of individual bacterial cells, with $80 \mathrm{~nm}$ resolution for the cell length, see Figure 6 (Kinnunen et al. 2010). Due to the superparamagnetic nature of the particles, equation (11) was used to measure how rotation-rate-changes translate into changes in effective volume of the sensor. The growth and division of individual Escherichia coli bacteria was observed, with $80-n m$ sensitivity to the cell length. Over the life cycle of a cell, up to a $300 \%$ increase was observed in the rotational period of the biosensor due to the increase in cell volume. In addition, we observed single bacterial cell growth response to antibiotics. This work demonstrates a non-microscopy based approach for monitoring individual cell growth dynamics, including cell elongation, generation time, lag time, and division, as well as the sensitivity to antibiotics.

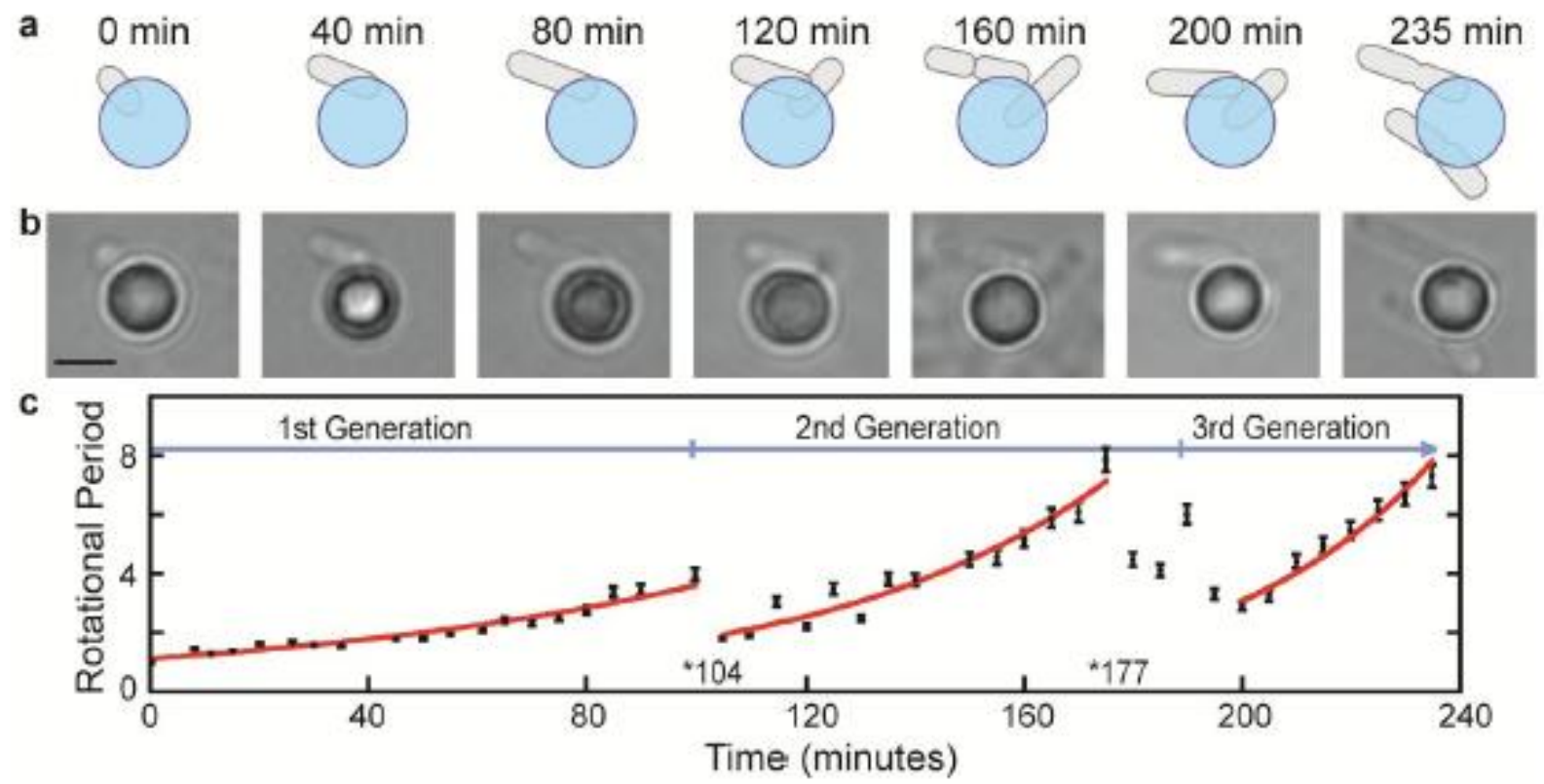

Figure 22: Growth and division of a single E. coli bacterium, measured with an AMBR sensor and observed with an optical microscope. (a) Schematic figures and (b) 100X oil immersion optical microscopy images of the magnetic particle sensor with initially a single bacterium attached and subsequent cell divisions. The scale bar is $2 \mu \mathrm{m}$. (c) Cell growth and division as observed with the AMBR sensor. After a period of growth, the first cell division is observed at 104 minutes and again at 177 and 199 minutes. The error bars correspond to the measurement error in the rotational period and the exponential fits are a guide to the eye. Data is normalized to 1 at time zero.

\section{High frequency AMBR}

Higher frequency asynchronous magnetic bead rotation allows better averaging, higher resolution and higher bandwidth studies, which will allow applications such as real time single bacterium growth monitoring with sub-diffraction limited sensitivity and single virus detection, both in their given fluid environment. The relative uncertainty in bead radius of the ferromagnetic AMBR sensor is proportional to the relative measurement uncertainty and has no explicit frequency dependence. The sensitivity of the system is therefore: 
$S=\frac{r}{3} \cdot \frac{\mathrm{D}\langle q\rangle}{\langle q\rangle}$,

where the sensitivity of the AMBR sensor, $S$, is defined as the smallest detectable change in the system's hydrodynamic radius $r$, and it is governed by the uncertainty in the bead frequency measurement, $\mathrm{D}\langle q\rangle$. In order to measure the uncertainty of the bead rotation measurement, a $6.7 \mu \mathrm{m}$ diameter magnetic bead AMBR sensor was driven with a $400 \mathrm{~Hz}, 1$ $\mathrm{mT}$, rotating magnetic field and was continuously measured over 37 seconds. The data is shown in Figure 23. The average rotation period was $25.4 \mathrm{~Hz}$, with a $0.7 \mathrm{~Hz}$ standard deviation. Using these values and equation (12) to calculate the sensitivity, the AMBR sensor was found to be sensitive to a $59 \mathrm{~nm}$ bead diameter change.

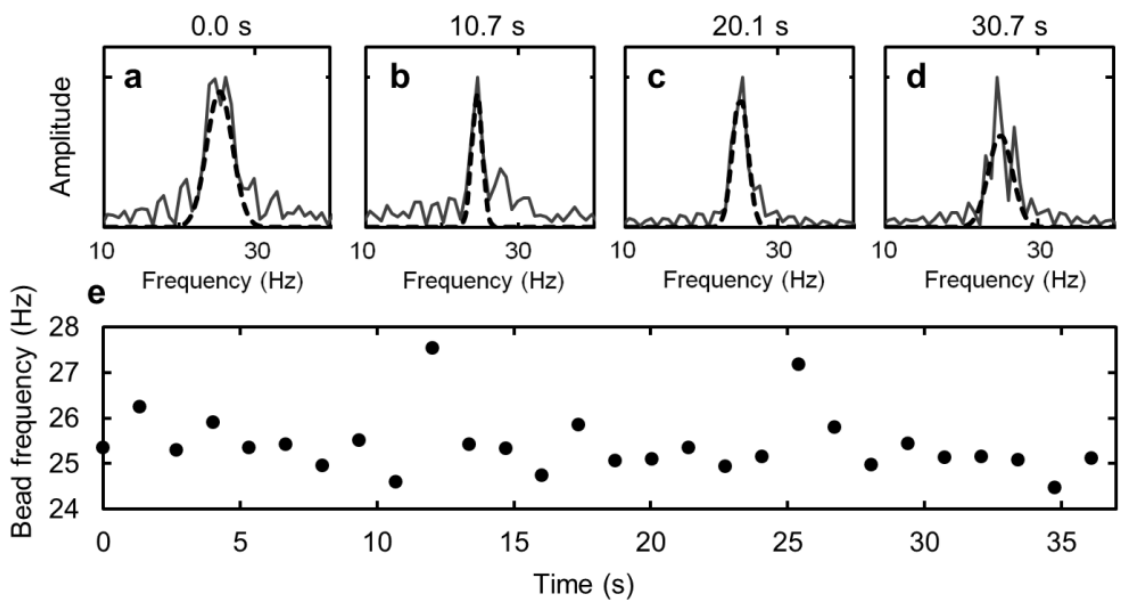

bead in $31^{\circ} \mathrm{C}$ water.
Figure 23: The AMBR sensor signal in time. (ad) Fast Fourier transforms of the rotation data, at time $0 \mathrm{~s}, 10.7 \mathrm{~s}, 20.1 \mathrm{~s}$ and $30.7 \mathrm{~s}$. (e) The rotational period of the AMBR sensor in time, as measured with the Fourier analysis, examples of which are shown in parts a-d. The average of the bead frequency is $25.4 \pm$ $0.7 \mathrm{~Hz}$. The AMBR sensor is a $6.7 \mu \mathrm{m}$ magnetic

\section{Microfluidic integration of AMBR}

Bead translation, magnetic interactions, surface adhesion and stiction are factors that may reduce the efficiency and accuracy of the AMBR biosensors under some experimental conditions (McNaughton et al. 2006). By confining individual AMBR sensors within nanolitersized water-in-oil (w/o) droplets in a microfluidic channel, the magnetic beads are isolated within a defined space, separated from the neighboring beads, and separated from the channel walls by a thin oil layer. Microfluidic platforms also reduce reagent use and enable parallelization and high throughput due to their small dimensions. Therefore, integration of AMBR with microfluidics is advantageous in many respects. Sinn et al. nanofabricated and characterized microfluidic channels to form droplets containing the maximum number of individual magnetic particles. The asynchronous rotation of the magnetic particles was characterized, concluding that water-in-oil droplets offer a good platform for AMBR studies with excellent stability and higher rotation frequencies compared to glass surface, see Figure 24 (Sinn et al. to be published). 
(a)

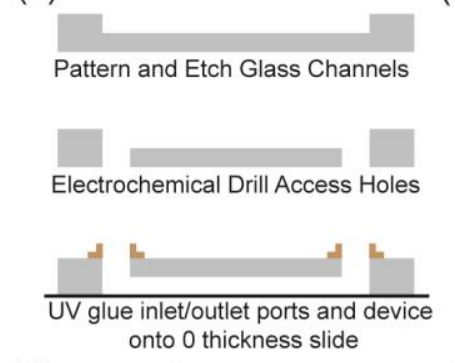

(d)

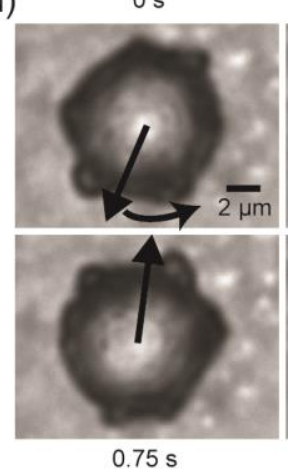

(b)

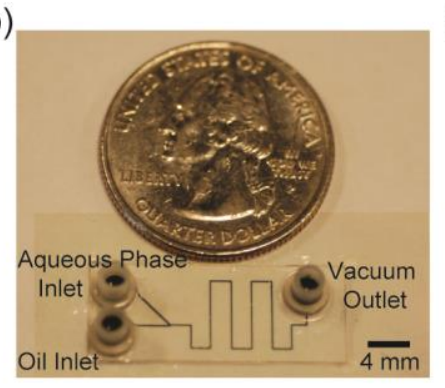

$0.25 \mathrm{~s}$

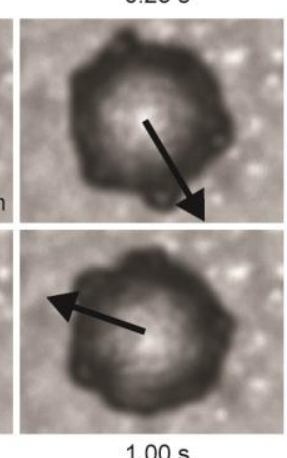

$0.50 \mathrm{~s}$

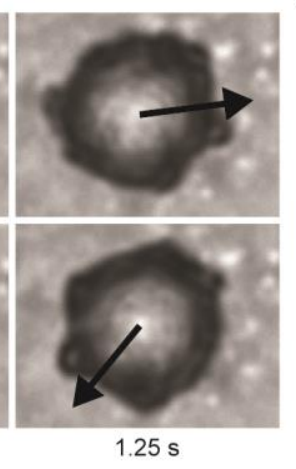

(e) (c)
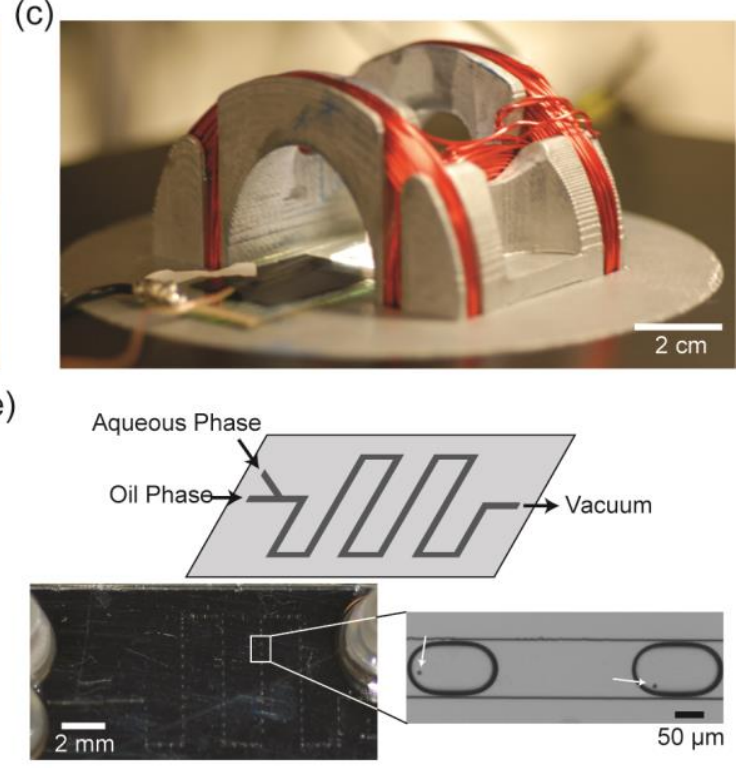

Figure 24: (a) Micro fabrication procedure. (b) Image of the microfluidic droplet device. (c) A picture of the microfluidic device on a microscope and inside the electromagnet coils, which generate a rotating magnetic field of $0.9 \mathrm{mT}$ amplitude at its core. (d) Optical microscopy image of an $8.8 \mu \mathrm{m}$ magnetic bead rotating asynchronously with an external rotating magnetic field with a $50 \mathrm{~Hz}$ driving frequency, bead rotation rate being much lower $(0.8 \mathrm{~Hz})$. Visual aid is provided to observe the bead rotation. (e) Droplets of $0.5 \mathrm{~nL}$ to $1 \mathrm{~nL}$ in volume are formed by applying vacuum at the outlet and applying hydrostatic pressure at the oil inlet. A microfluidic device with this design holds between 50 and 75 droplets.

\section{Small population growth and antibiotic susceptibility testing (AST)}

Inappropriate antibiotic use is a major factor contributing to the emergence and spread of antimicrobial resistance. The long turnaround time (over 24 hours) required for clinical antimicrobial susceptibility testing (AST) often results in patients being prescribed empiric therapies, which may be inadequate, inappropriate, or overly broad, prior to complete diagnosis. Reduction in the AST time would enable earlier and more appropriate therapies to be prescribed. A "Rapid AST" proof of principle study was done with AMBR biosensors in microfluidic water droplets, see Figure 9 (Sinn et al., to be published). AMBR biosensor droplet microfluidic platform was demonstrated, enabling single cell and small cell population growth studies for applications aimed towards rapid AST. A small cell population of uropathogenic $E$. coli was confined in microfluidic droplets and exposed to concentrations above and below the minimum inhibitory concentration (MIC) of gentamicin. Growth was observed for bacteria treated with gentamicin concentrations below the MIC; no growth was observed for bacteria treated with gentamicin concentrations above the MIC. A $100 \%$ difference in the sensor signal (ie. rotational period), was observed within 25 minutes between samples with and without antibiotics. 


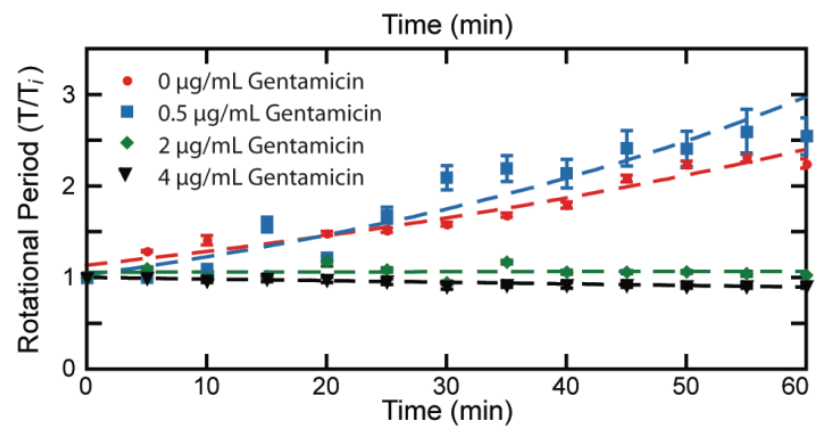

Figure 25: Small population E. coli growth curves measured with AMBR biosensors. The MIC is $1 \mu \mathrm{g} / \mathrm{mL}$. Bacteria treated with gentamicin concentrations below the MIC continued to grow, whereas bacteria treated with concentrations above the MIC did not.

\section{Off-the-microscope studies}

The ability of the AMBR biosensors to transform femtoliter scale volume changes into frequency variations of orders-of-magnitude-larger magnetic particles is what makes the technology powerful. While most of the AMBR biosensor experiments to date have been conducted on an optical microscope, it is not ideal for automation purposes. The rotation rate of the AMBR sensor can be measured by its vertical displacement over time (see Figure 26) (McNaughton et al. 2006), or by focusing a low power laser, which has been done using a simple prototype device without optical microscope (Kinnunen et al. 2010; McNaughton et al. 2009). A fast Fourier transform (FFT) can be used to decipher the rotation rate of a particle from the periodic raw signal, for example the displacement of the particle or the laser intensity fluctuating due to particle rotation. Using the above mentioned methods, the work is underway to improve AMBR biosensor compatibility with the industry standard 96and 384-well plates for rapid growth studies such as antimicrobial susceptibility testing (AST) of bacteria, chemotherapy sensitivity and resistance assays (CSRAs) for cancer cells, yeast antifungal testing and research applications, where nano- or microscale growth have to be rapidly measured.

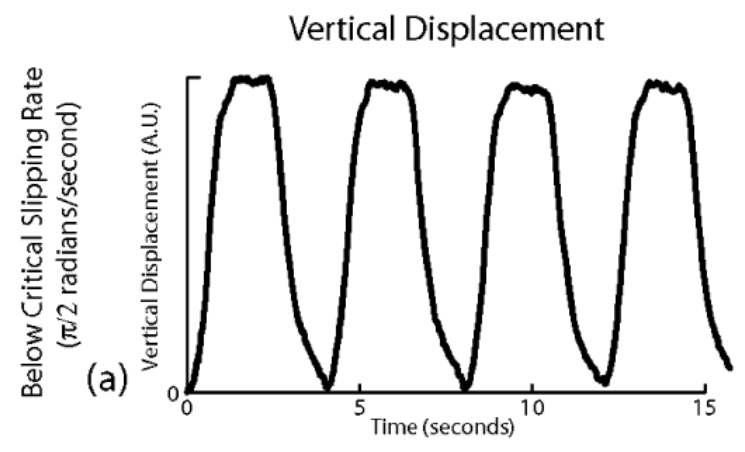

\section{Fourier Transform (Average Rotation Rate)}
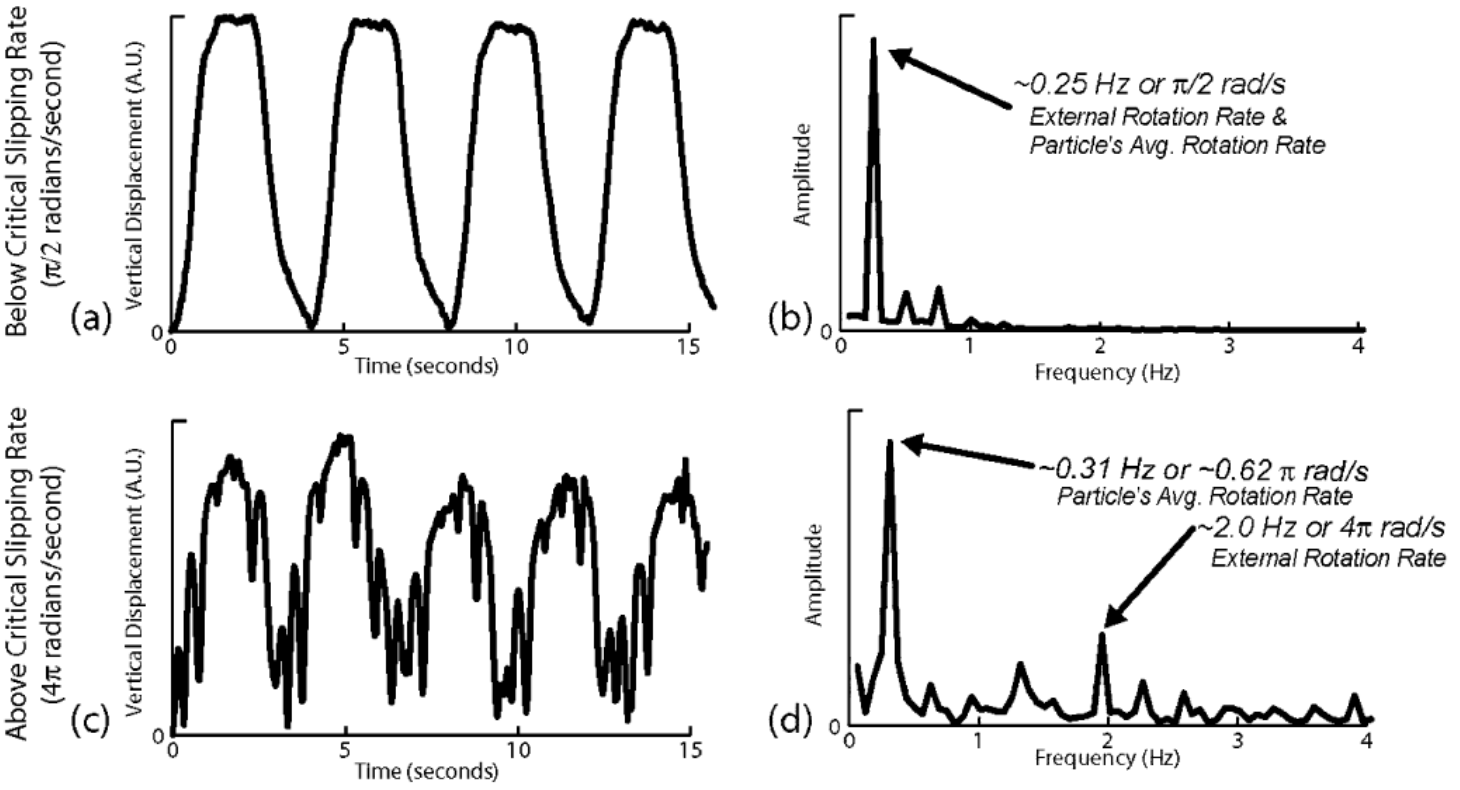

Figure 26: The rotational behavior of a single magnetic particle below (a-b) and above (c-d) the critical driving frequency. A Fourier transform was applied to the periodic data to determine the 
average rotation rate, parts $b$ and $d$. Below the critical driving frequency, part b shows only one main peak, which is a result of the particle being in phase with the external field (with a constant phase lag). At external rates above the critical frequency, part $d$ reveals two main peaks, one that results from the slower net rotation of the particle and one that results from the faster counterclockwise rocking of the magnetic particle.

\section{Cell magneto-rotation ( $\mathrm{CM}$ ) and cancer cell studies}

Suspended cells behave differently than adherent cells, which is especially important in predicting the environmental responses of circulating tumor cells (CTCs) and stem cells. However, due to the lack of existing methods, the growth response of cancer cells in the suspended state is difficult to study. Elbez et al. (2010) describe a new method for quantitative, real time monitoring of cell size and morphology, on individual live suspended cancer cells, unconfined in three dimensions. The precision is comparable to that of the best optical microscopes, but there is no need for confining the cell to the imaging plane. The cell magneto-rotation (CM) method is based on nanoparticle induced cell magnetization of the cell, where magnetic nanoparticles are attached to the cell or internalized in the cytoplasm. By using a rotating magnetic field, the magnetically labeled cells are actively rotated, and the rotational period can be measured in real-time. The theory behind the cell magnetorotation is analogous to the SPM particle rotation where magnetic nanoparticles are embedded inside a nonmagnetic core; see Equations (6) - (10). In the case of CM, the nonmagnetic core is replaced with a living cell and the magnetic nanoparticles are replaced with functionalized magnetic nanoparticles. A change in morphology induces a change in the rotational period of the suspended cell (e.g. a bigger cell rotates slower). Using CM it is possible to monitor cell swelling and death, in real time at the single cell level, see Figure 27. This method could be used for multiplexed real time single cell morphology analysis, with implications for СTC drug sensitivity, drug testing, drug discovery, genomics and threedimensional culturing (Elbez et al. 2010).

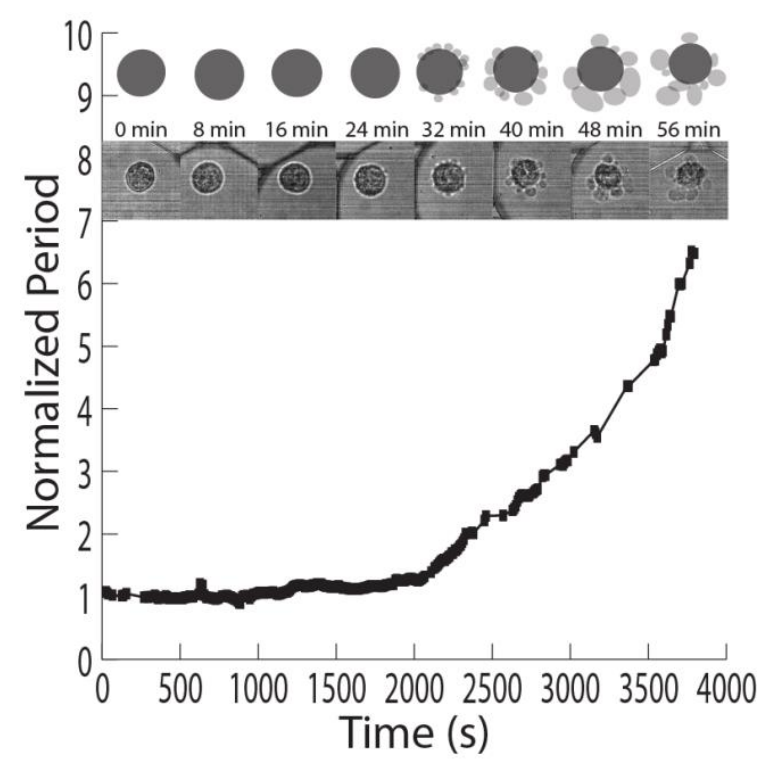

Figure 27: Changes in the rotation period of a single live HeLa cell in $5 \%$ Ethanol. The bottom pictures show snapshots of the rotated cell at each indicated time, while the schematic pictures on top show the corresponding cell shapes. Dark discs represent the cell cytoplasm and membrane, while grey spots show the vesicles formed at the surface. Note the 550\% increase in rotation period due to cell "blebbing".

\section{Label-acquired magneto-rotation (LAM) biosensor}

Another type of AMBR based biosensor has been demonstrated, which takes advantage of the fact that the rotation rate depends on the amount of magnetic material present in the 
particle (see equations (6) - (10)) (Hecht et al. 2011). Instead of measuring volume (and assuming constant magnetic moment), the label-acquired magneto-rotation (LAM) biosensor measures the magnetic content (and assumes the volume to be constant). Therefore, when a nonmagnetic sphere acquires magnetic moment by specific antigenantibody interactions, the amount of antigen in the sample is reflected by the acquired magnetic moment and therefore the rotation rate of the particle. See Figure 28 for a schematic of the system. LAM biosensor has been used to detect Thrombin detection limit below $1 \mathrm{nM}$.

(a)

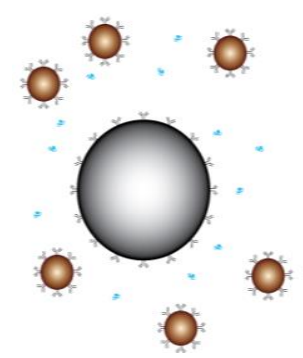

Step 2: Binding

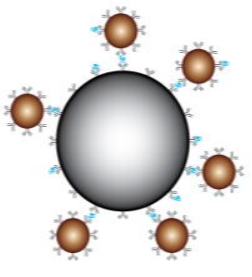

Step 3: Rotation

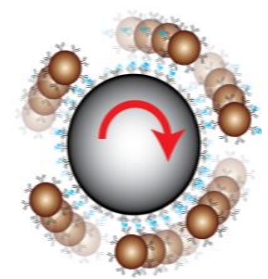

(b)

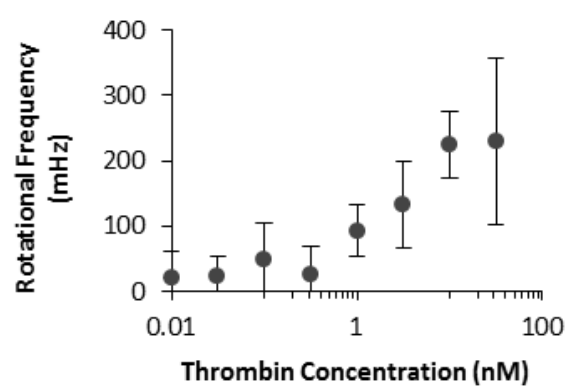

Figure 28: a) A schematic illustration of the label-acquired magnetorotation. A central $10 \mu \mathrm{m}$ nonmagnetic sphere and $1 \mu \mathrm{m}$ superparamagnetic label beads are coated with a sandwich pair of affinity molecules (either antibodies or aptamers) specific to the target, and are then mixed with the target. The target is sandwiched between the central sphere and the label beads, creating a magnetic sandwich complex. The sandwich complex is transferred to a rotating magnetic field, where the rotational frequency of the sandwich complex is a function of the number of attached superparamagnetic label beads (and therefore the concentration of the target as well). In the absence of the target, a sandwich complex is not formed, so the nonmagnetic central spheres do not rotate in the magnetic field. b) An example of the application of label-acquired magnetorotation. Thrombin was chosen as a sample analyte and detected using a sandwich aptamer pair.

\section{Magnetically modulated optical nanoprobes}

Modulated optical nanoprobes (MOONs) can be nonmagnetic, modulated by Brownian rotation (Behrend, Anker, and Kopelman 2004), or magnetic, modulated with rotating magnetic fields (MagMOONs) (Anker, Behrend, and Kopelman 2003; Anker and Kopelman 2003). The periodic magnetic modulation of the MagMOONs can be used to separate a weak fluorescent signal from the background fluorescence (Figure 29). 
a)

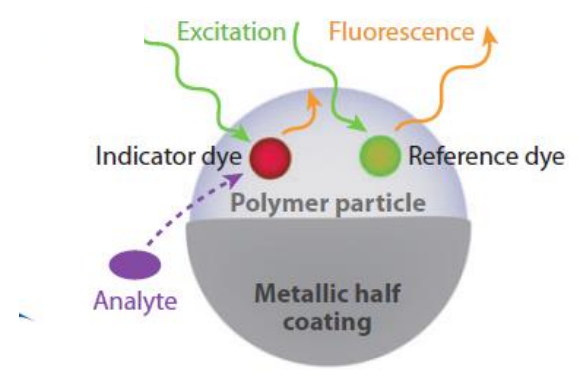

b)
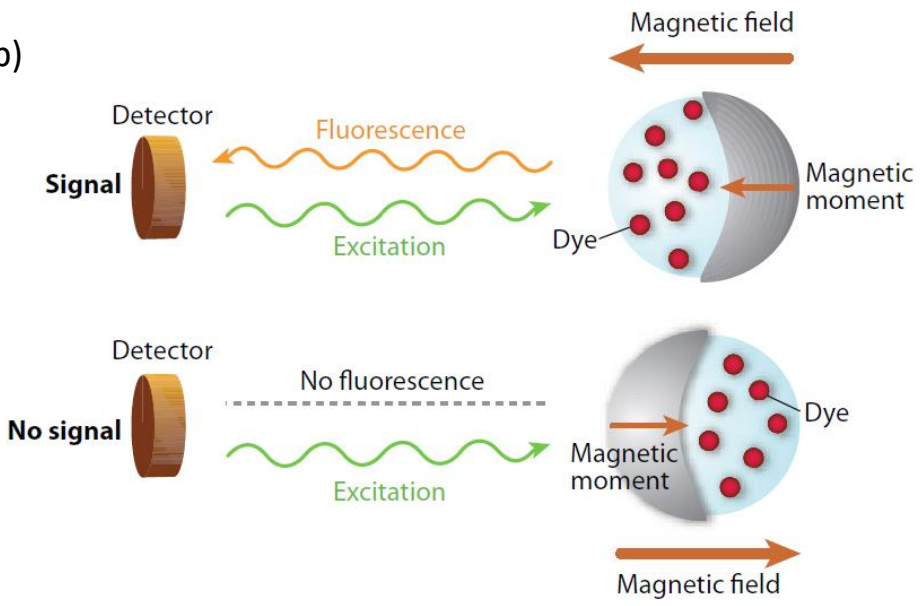

Figure 29: (a) Modulated optical nanoprobe sensor. (b) Background-free measurement taken by a magnetically modulated optical nanoprobe (MagMOON). An external magnetic field orients the MagMOON, causing its fluorescent excitation and observed emission to blink on and off as it rotates. Note that the background fluorescence does not blink.

This simple procedure increases the signal-to-background ratio by 3-4 orders of magnitude (Anker and Kopelman 2003), enabling the use of fluorescent nanoparticle sensors in samples with highly scattering or fluorescent backgrounds. The MOON functionality can be added to any fluorescent nanoparticle sensor by using a vacuum deposition with magnetic or nonmagnetic metal as the coating material. The MOONs have also been used for local viscosity measurements by using the AMBR technique (McNaughton, Agayan, Wang et al. 2007), which enables simultaneous physical and chemical measurements if fluorescent probes are used. Note that for background subtraction applications it is preferable to rotate the MOONs synchronously with the external rotating magnetic field, not asynchronously as is done with the AMBR sensor applications.

\section{References:}

Agrawal, Amit, Tushar Sathe, and Shuming Nie. 2007. Single-Bead Immunoassays Using Magnetic Microparticles and Spectral-Shifting Quantum Dots. Journal of Agricultural and Food Chemistry 55 (10):3778-3782 \%U http://dx.doi.org/10.1021/jf0635006.

Anker, J. N., C. J. Behrend, H. Huang, and R. Kopelman. 2005. Magnetically-modulated optical nanoprobes (MagMOONs) and systems. Journal of Magnetism and Magnetic Materials 293 (1):655-662.

Anker, J. N., C. Behrend, and R. Kopelman. 2003. Aspherical magnetically modulated optical nanoprobes (MagMOONs). Journal of Applied Physics 93:6698.

Anker, J. N., and R. Kopelman. 2003. Magnetically modulated optical nanoprobes. Applied Physics Letters 82 (7):1102 \%U http://link.aip.org/link/APPLAB/v82/i7/p1102/s1\&Agg=doi.

Aytur, Turgut, Jonathan Foley, Mekhail Anwar, Bernhard Boser, Eva Harris, and P. Robert Beatty. 2006. A novel magnetic bead bioassay platform using a microchip-based sensor for infectious disease diagnosis. Journal of Immunological Methods 314 (12):21-29\%U http://www.sciencedirect.com/science/article/B6T2Y-4KB107N1/2/1e915e52002942b0d7a8fbbb699886ae. 
Bacri, J. C., A. Cēbers, and R. Perzynski. 1994. Behavior of a magnetic fluid microdrop in a rotating magnetic field. Physical Review Letters 72 (17):2705 \%U

http://link.aps.org/doi/10.1103/PhysRevLett.72.2705.

Bao, Y. P., T. F. Wei, P. A. Lefebvre, H. An, L. He, G. T. Kunkel, and U. R. M\üller. 2006. Detection of protein analytes via nanoparticle-based bio bar code technology. Anal. Chem 78 (6):2055-2059.

Baselt, David R., Gil U. Lee, Mohan Natesan, Steven W. Metzger, Paul E. Sheehan, and Richard J. Colton. 1998. A biosensor based on magnetoresistance technology. Biosensors and Bioelectronics 13 (7-8):731-739 \%U http://www.sciencedirect.com/science/article/B6TFC-3V7468X2/2/2062aec052caa896da42da32714f13ee.

Behrend, C. J., J. N. Anker, and R. Kopelman. 2004. Brownian modulated optical nanoprobes. Applied Physics Letters 84 (1):154 \%U http://link.aip.org/link/APPLAB/v84/i1/p154/s1\&Agg=doi.

Besse, Pierre- A., Giovanni Boero, Michel Demierre, Vincent Pott, and Radivoje Popovic. 2002. Detection of a single magnetic microbead using a miniaturized silicon Hall sensor. Applied Physics Letters 80 (22):4199\%U http://link.aip.org/link/APPLAB/v80/i22/p4199/s1\&Agg=doi.

Biswal, S. L., and A. P. Gast. 2004. Micromixing with linked chains of paramagnetic particles. Analytical Chemistry 76 (21):6448.

Boisen, A., and T. Thundat. 2009. Design \& fabrication of cantilever array biosensors. Materials Today 12 (9):32-38.

Brehm-Stecher, B. F., and E. A. Johnson. 2004. Single-cell microbiology: tools, technologies, and applications. Microbiology and Molecular Biology Reviews 68 (3): 538 .

Bryan, A. K., A. Goranov, A. Amon, and S. R. Manalis. 2010. Measurement of mass, density, and volume during the cell cycle of yeast. Proceedings of the National Academy of Sciences 107 (3): 999.

Brzeska, M., M. Panhorst, P. B. Kamp, J. Schotter, G. Reiss, A. P\ühler, A. Becker, and H. Br\ückl. 2004. Detection and manipulation of biomolecules by magnetic carriers. Journal of biotechnology 112 (1-2):25-33.

Cai, Sheng, Liang Xin, Choiwan Lau, and Jianzhong Lu. 2010. Highly sensitive non-stripping gold nanoparticles-based chemiluminescent detection of DNA hybridization coupled to magnetic beads. The Analyst 135 (3):615 \%U http://pubs.rsc.org/en/Content/ArticleLanding/2010/AN/b927359j.

Caroli, C., and P. Pincus. 1969. Response of an isolated magnetic grain suspended in a liquid to a rotating field. Physik der Kondensierten Materie 9 (4):311-319 \%U http://www.springerlink.com/content/a76712t6h6620w37/.

Chemla, Y. R., H. L. Grossman, Y. Poon, R. McDermott, R. Stevens, M. D. Alper, and J. Clarke. 2000. Ultrasensitive magnetic biosensor for homogeneous immunoassay. Proceedings of the National Academy of Sciences of the United States of America 97 (26): 14268 -14272 \%U http://www.pnas.org/content/97/26/14268.abstract.

D'Orazio, P. 2003. Biosensors in clinical chemistry. Clinica Chimica Acta 334 (1-2):41-69.

Dieny, B., V. S. Speriosu, S. Metin, S. S. P. Parkin, B. A. Gurney, P. Baumgart, and D. R. Wilhoit. 1991. Magnetotransport properties of magnetically soft spin-valve structures (invited). Journal of Applied Physics 69 (8):4774 \%U http://link.aip.org/link/JAPIAU/v69/i8/p4774/s1\&Agg=doi.

Edelstein, R. L., C. R. Tamanaha, P. E. Sheehan, M. M. Miller, D. R. Baselt, L. J. Whitman, and R. J. Colton. 2000. The BARC biosensor applied to the detection of biological warfare agents. Biosensors and Bioelectronics 14 (10-11):805-813.

Ejsing, L., M. F. Hansen, A. K. Menon, H. A. Ferreira, D. L. Graham, and P. P. Freitas. 2004. Planar Hall effect sensor for magnetic micro-and nanobead detection. Applied Physics Letters 84:4729. 
Elbez, R., B. H. McNaughton, L. Patel, J. K. Pienta, and R. Kopelman. 2010. Nanoparticle induced Cell Magneto-Rotation: Monitoring Morphology, Stress and Drug Sensitivity of a Suspended Single Cancer Cell.

Elfwing, A., Y. LeMarc, J. Baranyi, and A. Ballagi. 2004. Observing growth and division of large numbers of individual bacteria by image analysis. Applied and Environmental Microbiology 70 (2):675.

Enpuku, K., K. Inoue, K. Soejima, K. Yoshinaga, H. Kuma, and N. Hamasaki. 2005. Magnetic immunoassays utilizing magnetic markers and a high-Tc SQUID. Applied Superconductivity, IEEE Transactions on 15 (2):660-663.

Enpuku, K., K. Soejima, T. Nishimoto, T. Matsuda, H. Tokumitsu, T. Tanaka, K. Yoshinaga, H. Kuma, and N. Hamasaki. 2007. Biological Immunoassays Without Bound/Free Separation Utilizing Magnetic Marker and HTS SQUID. Applied Superconductivity, IEEE Transactions on 17 (2):816-819.

Erdem, Arzum, M. Isabel Pividori, Anabel Lermo, Alessandra Bonanni, Manel del Valle, and Salvador Alegret. 2006. Genomagnetic assay based on label-free electrochemical detection using magneto-composite electrodes. Sensors and Actuators B: Chemical 114 (2):591-598 \%U http://apps.isiknowledge.com/CitedFullRecord.do?product=UA\&db id=WOS\&SID =4B L5L1G2C26cdpkcA@9\&search mode=CitedFullRecord\&isickref=149670028.

Erglis, Kaspars, Qi Wen, Velta Ose, Andris Zeltins, Anatolijs Sharipo, Paul A. Janmey, and A. Cebers. 2007. Dynamics of Magnetotactic Bacteria in a Rotating Magnetic Field. Biophysical Journal 93 (4):1402-1412 \%U http://www.sciencedirect.com/science/article/B94RW-4TVR65716/2/c62b22673111b3e4ab06269631311f2f.

Fannin, P. C., L. Cohen-Tannoudji, E. Bertrand, A. T. Giannitsis, C. Mac Oireachtaigh, and J. Bibette. 2006. Investigation of the complex susceptibility of magnetic beads containing maghemite nanoparticles. Journal of Magnetism and Magnetic Materials 303 (1):147-152 \%U http://www.sciencedirect.com/science/article/B6TJJ-4HRN3H61/2/700aba01bb615b272c06e0a46e40b101.

Ferapontova, Elena E., Majken N. Hansen, Aaron M. Saunders, Stepan Shipovskov, Duncan S. Sutherland, and Kurt V. Gothelf. 2010. Electrochemical DNA sandwich assay with a lipase label for attomole detection of DNA. Chemical Communications 46 (11):1836 $\%$ Uttp://xlink.rsc.org/?DOI=b924627d.

Ferreira, H. A., D. L. Graham, P. P. Freitas, and J. M. S. Cabral. 2003. Biodetection using magnetically labeled biomolecules and arrays of spin valve sensors (invited). Journal of Applied Physics 93:7281.

Fritz, Jürgen. 2008. Cantilever biosensors. The Analyst 133 (7):855 \%U http://apps.isiknowledge.com/full record.do?product=UA\&search mode=Refine\&qid $=58 \&$ SID=4Cae37FbIfNh@p1kGij\&page =2\&doc=14.

Gaster, R. S., D. A. Hall, C. H. Nielsen, S. J. Osterfeld, H. Yu, K. E. Mach, R. J. Wilson, B. Murmann, J. C. Liao, S. S. Gambhir, and others. 2009. Matrix-insensitive protein assays push the limits of biosensors in medicine. Nature Medicine:1327-1332.

Gaub, H. E., and H. M. McConnell. 1986. Shear viscosity of monolayers at the air-water interface. The Journal of Physical Chemistry 90 (26):6830-6832 \%U http://dx.doi.org/10.1021/j100284a024.

Gehring, Andrew G., C. Gerald Crawford, Ralph S. Mazenko, Lisa J. Van Houten, and Jeffrey D. Brewster. 1996. Enzyme-linked immunomagnetic electrochemical detection of Salmonella typhimurium. Journal of Immunological Methods 195 (1-2):15-25\%U http://www.sciencedirect.com/science/article/B6T2Y-3W3169C3/2/08f8285b691b402c1ba46ed51403d678.

Ghosh, A., and P. Fischer. 2009. Controlled propulsion of artificial magnetic nanostructured propellers. Nano Letters 9 (6):2243-2245. 
Graham, D. L., H. A. Ferreira, N. Feliciano, P. P. Freitas, L. A. Clarke, and M. D. Amaral. 2005. Magnetic field-assisted DNA hybridisation and simultaneous detection using micron-sized spin-valve sensors and magnetic nanoparticles. Sensors and Actuators B: Chemical 107 (2):936-944.

Graham, D. L., H. A. Ferreira, and P. P. Freitas. 2004. Magnetoresistive-based biosensors and biochips. TRENDS in Biotechnology 22 (9):455-462.

Grancharov, S. G., H. Zeng, S. Sun, S. X. Wang, S. O'Brien, C. B. Murray, J. R. Kirtley, and G. A. Held. 2005. Bio-functionalization of monodisperse magnetic nanoparticles and their use as biomolecular labels in a magnetic tunnel junction based sensor. J. Phys. Chem. B 109 (26):13030-13035.

Grimm, Jan, J. Manuel Perez, Lee Josephson, and Ralph Weissleder. 2004. Novel Nanosensors for Rapid Analysis of Telomerase Activity. Cancer Research 64 (2):639 $643 \%$ Uttp://cancerres.aacrjournals.org/content/64/2/639.abstract.

Gupta, A. K., and M. Gupta. 2005. Synthesis and surface engineering of iron oxide nanoparticles for biomedical applications. Biomaterials 26 (18):3995-4021.

Gupta, A. K., R. R. Naregalkar, V. D. Vaidya, and M. Gupta. 2007. Recent advances on surface engineering of magnetic iron oxide nanoparticles and their biomedical applications. Nanomedicine 2 (1):23-39.

Gusev, Yuriy, Jason Sparkowski, Arumugham Raghunathan, Harley Ferguson, Jane Montano, Nancy Bogdan, Barry Schweitzer, Steven Wiltshire, Stephen F. Kingsmore, Warren Maltzman, and Vanessa Wheeler. 2001. Rolling Circle Amplification. The American Journal of Pathology 159 (1):63-69.

Haun, Jered B., Tae-Jong Yoon, Hakho Lee, and Ralph Weissleder. 2010. Magnetic nanoparticle biosensors. Wiley Interdisciplinary Reviews: Nanomedicine and Nanobiotechnology:n/a-n/a \%U http://onlinelibrary.wiley.com/doi/10.1002/wnan.84/pdf.

Hecht, A., P. Kinnunen, B. H. McNaughton, and R. Kopelman. 2011. Label-acquired magnetorotation for biosensing: An asynchronous rotation assay. Journal of Magnetism and Magnetic Materials 323 (3-4):272-278\%U http://www.sciencedirect.com/science/article/B6TJJ-512VTP75/2/63c6ad753d8fa40cf0d89ed032d87db2.

Heim, D. E., R. E. Fontana, C. Tsang, V. S. Speriosu, B. A. Gurney, and M. L. Williams. 1994. Design and operation of spin valve sensors. Magnetics, IEEE Transactions on 30 (2):316-321.

Helgesen, Geir, Piotr Pieranski, and Arne T. Skjeltorp. 1990. Nonlinear phenomena in systems of magnetic holes. Physical Review Letters 64 (12):1425 \%U http://link.aps.org/doi/10.1103/PhysRevLett.64.1425.

Hill, H. D., and C. A. Mirkin. 2006. The bio-barcode assay for the detection of protein and nucleic acid targets using DTT-induced ligand exchange. Nature Protocols 1 (1):324336.

Hill, H. D., R. A. Vega, and C. A. Mirkin. 2007. Nonenzymatic detection of bacterial genomic DNA using the bio bar code assay. Anal. Chem 79 (23):9218-9223.

Honda, T., K. I. Arai, K. Ishiyama, and others. 1996. Micro swimming mechanisms propelled by external magnetic fields. IEEE Transactions on Magnetics 32 (5):5085-5087.

Hsing, I., Xu Ying, and Zhao Wenting. 2007. Micro-and Nano-Magnetic Particles for Applications in Biosensing. Electroanalysis 19 (7-8):755-768.

Inoue, I., Y. Wakamoto, H. Moriguchi, K. Okano, and K. Yasuda. 2001. On-chip culture system for observation of isolated individual cells. Lab on a Chip 1 (1):50-55.

Janssen, X. J. A., A. J. Schellekens, K. van Ommering, L. J. van Ijzendoorn, and M. W. J. Prins. 2009. Controlled torque on superparamagnetic beads for functional biosensors. Biosensors and Bioelectronics 24 (7):1937-1941. 
Jiang, Z., J. Llandro, T. Mitrelias, and J. A. C. Bland. 2006. An integrated microfluidic cell for detection, manipulation, and sorting of single micron-sized magnetic beads. Journal of Applied Physics 99:08S105.

Jorgensen, J. H., and M. J. Ferraro. 1998. Antimicrobial Susceptibility Testing: General Principles and Contemporary Practices. Clinical Infectious Diseases 26 (4):973-980 \%U http://www.jstor.org/stable/4481507.

- - . 2009. Antimicrobial susceptibility testing: a review of general principles and contemporary practices. Clinical Infectious Diseases: An Official Publication of the Infectious Diseases Society of America 49 (11):1749-1755 \%U http://www.ncbi.nlm.nih.gov/pubmed/19857164.

Kaittanis, Charalambos, Sudip Nath, and J. Manuel Perez. 2008. Rapid NanoparticleMediated Monitoring of Bacterial Metabolic Activity and Assessment of Antimicrobial Susceptibility in Blood with Magnetic Relaxation. PLoS ONE 3 (9):e3253 \%U http://dx.doi.org/10.1371/journal.pone.0003253.

Kaittanis, Charalambos, Santimukul Santra, and J. Manuel Perez. 2009. Role of Nanoparticle Valency in the Nondestructive Magnetic-Relaxation-Mediated Detection and Magnetic Isolation of Cells in Complex Media. Journal of the American Chemical Society 131 (35): $12780-12791 \% U$ http://apps.isiknowledge.com/full record.do?product=UA\&colname=WOS\&search m ode=CitingArticles\&qid =11\&SID=4BL5L1G2C26cdpkcA@9\&page=1\&doc $=2$.

Kasatkin, S. I., N. P. Vasil'eva, and A. M. Murav'ev. 2010. Biosensors based on the thin-film magnetoresistive sensors. Automation and Remote Control 71 (1):156-166.

Katz, Eugenii, Itamar Willner, and Joseph Wang. 2004. Electroanalytical and Bioelectroanalytical Systems Based on Metal and Semiconductor Nanoparticles. Electroanalysis 16 (1-2):19-44 \%U http://dx.doi.org/10.1002/elan.200302930.

Kiely, J., P. Hawkins, P. Wraith, and R. Luxton. 2007. Paramagnetic particle detection for use with an immunoassay based biosensor. Science, Measurement \& Technology, IET $1(5): 270-275$.

Kim, Gha-Young, and Ahjeong Son. 2010. Development and characterization of a magnetic bead-quantum dot nanoparticles based assay capable of Escherichia coli O157:H7 quantification. Analytica Chimica Acta 677 (1):90-96 \%U http://www.sciencedirect.com/science/article/B6TF4-50PVG3B3/2/c89c572c205641723e4b2683d6eaec77.

Kinnunen, P., I. Sinn, B. H. McNaughton, and R. Kopelman. 2010. High frequency asynchronous magnetic bead rotation for improved biosensors. Applied Physics Letters 97 (22): $223701 \%$ U http://link.aip.org.proxy.lib.umich.edu/link/APPLAB/v97/i22/p223701/s1\&Agg=doi.

Korneva, G., H. Ye, Y. Gogotsi, D. Halverson, G. Friedman, J. C. Bradley, and K. G. Kornev. 2005. Carbon nanotubes loaded with magnetic particles. Nano Letters 5 (5):879884.

Kotitz, R., H. Matz, L. Trahms, H. Koch, W. Weitschies, T. Rheinlander, W. Semmler, and T. Bunte. 1997. SQUID based remanence measurements for immunoassays. Applied Superconductivity, IEEE Transactions on 7 (2):3678-3681.

Krishnan, K. M. 2010. Biomedical Nanomagnetics: A Spin Through Possibilities in Imaging, Diagnostics, and Therapy. IEEE transactions on magnetics 46 (7):2523-2558.

Kriz, C. B., K. Radevik, and D. Kriz. 1996. Magnetic permeability measurements in bioanalysis and biosensors. Anal. Chem 68 (11):1966-1970.

Kriz, K., J. Gehrke, and D. Kriz. 1998. Advancements toward magneto immunoassays. Biosensors and Bioelectronics 13 (7-8):817-823.

Kriz, K., F. Ibraimi, M. Lu, L. O. Hansson, and D. Kriz. 2005. Detection of C-reactive protein utilizing magnetic permeability detection based immunoassays. Anal. Chem 77 (18):5920-5924. 
Kuma, Hiroyuki, Hiroko Oyamada, Akira Tsukamoto, Takako Mizoguchi, Akihiko Kandori, Yoshinori Sugiura, Kohji Yoshinaga, Keiji Enpuku, and Naotaka Hamasaki. 2010. Liquid phase immunoassays utilizing magnetic markers and SQUID magnetometer. Clinical Chemistry and Laboratory Medicine 48 (9):1263-1269 \%U

Kuramitz, Hideki. 2009. Magnetic microbead-based electrochemical immunoassays. Analytical and Bioanalytical Chemistry 394 (1):61-69 \%U http://dx.doi.org/10.1007/s00216-009-2650-y.

Lācis, S., J. C. Bacri, A. Cēbers, and R. Perzynski. 1997. Frequency locking and devil's staircase for a two-dimensional ferrofluid droplet in an elliptically polarized rotating magnetic field. Physical Review E 55 (3):2640 \%U http://link.aps.org/doi/10.1103/PhysRevE.55.2640.

Lea, T., F. Vartdal, K. Nustad, S. Funderud, A. Berge, T. Ellingsen, R. Schmid, P. Stenstad, and J. Ugelstad. 1988. Monosized, magnetic polymer particles: Their use in separation of cells and subcellular components, and in the study of lymphocyte function <I > in vitro</I >. Journal of Molecular Recognition 1 (1):9-18 \%U http://dx.doi.org/10.1002/jmr.300010104.

Li, G., V. Joshi, R. L. White, S. X. Wang, J. T. Kemp, C. Webb, R. W. Davis, and S. Sun. 2003. Detection of single micron-sized magnetic bead and magnetic nanoparticles using spin valve sensors for biological applications. Journal of Applied Physics 93:7557.

Li, G., S. Sun, R. J. Wilson, R. L. White, N. Pourmand, and S. X. Wang. 2006. Spin valve sensors for ultrasensitive detection of superparamagnetic nanoparticles for biological applications. Sensors and Actuators A: Physical 126 (1):98-106.

Lim, Sun Hee, Felix Bestvater, Philippe Buchy, Sek Mardy, and Alexey Dan Chin Yu. 2009. Quantitative Analysis of Nucleic Acid Hybridization on Magnetic Particles and Quantum Dot-Based Probes. Sensors 9 (7):5590-5599 \%U http://apps.isiknowledge.com/full record.do?product=UA\&colname=WOS\&search $\mathrm{m}$ ode=CitingArticles\&qid=26\&SID=4Cae37FbIfNh@p1kGij\&page =1\&doc=6.

Liu, Guodong, Joseph Wang, Jeonghwan Kim, M. Rasul Jan, and Greg E. Collins. 2004. Electrochemical Coding for Multiplexed Immunoassays of Proteins. Analytical Chemistry 76 (23):7126-7130 \%U http://dx.doi.org/10.1021/ac049107l.

Liu, Y., D. Yao, H. Chang, C. Liu, and C. Chen. 2008. Magnetic bead-based DNA detection with multi-layers quantum dots labeling for rapid detection of Escherichia coli 0157:H7. Biosensors and Bioelectronics 24 (4):558-565 \%U http://apps.isiknowledge.com/full record. do?product=UA\&search mode $=$ GeneralSea rch\&qid=1\&SID=4DCMgl@oDdE2BD24kJk\&page=1\&doc=1\&colname=WOS.

Loureiro, J., R. Ferreira, S. Cardoso, P. P. Freitas, J. Germano, C. Fermon, G. Arrias, M. Pannetier-Lecoeur, F. Rivadulla, and J. Rivas. 2009. Toward a magnetoresistive chip cytometer: Integrated detection of magnetic beads flowing at $\mathrm{cm} / \mathrm{s}$ velocities in microfluidic channels. Applied Physics Letters 95:034104.

McNaughton, B. H., R. R. Agayan, R. Clarke, R. G. Smith, and R. Kopelman. 2007. Single bacterial cell detection with nonlinear rotational frequency shifts of driven magnetic microspheres. Applied Physics Letters 91:224105.

McNaughton, B. H., R. R. Agayan, J. X. Wang, and R. Kopelman. 2007. Physiochemical microparticle sensors based on nonlinear magnetic oscillations. Sensors \& Actuators: B. Chemical 121 (1):330-340.

McNaughton, B. H., J. N. Anker, and R. Kopelman. 2005. Magnetic microdrill as a modulated fluorescent pH sensor. Journal of Magnetism and Magnetic Materials 293 (1):696-701 \%U http://www.sciencedirect.com/science/article/B6TJJ-4FTS0766/2/5392ee1f14f984193660a388cd24db45.

McNaughton, B. H., K. A. Kehbein, J. N. Anker, and R. Kopelman. 2006. Sudden breakdown in linear response of a rotationally driven magnetic microparticle and application to 
physical and chemical microsensing. Journal of Physical Chemistry B 110 (38): 18958.

McNaughton, B. H., P. Kinnunen, R. G. Smith, S. N. Pei, R. Torres-Isea, R. Kopelman, and R. Clarke. 2009. Compact sensor for measuring nonlinear rotational dynamics of driven magnetic microspheres with biomedical applications. Journal of Magnetism and Magnetic Materials 321 (10):1648-1652 \%U http://www.sciencedirect.com/science/article/B6TJ]-4VNKGYCN/2/0a4b577717a9a7e2c9e779c7d5d93465.

Megens, M., and M. Prins. 2005. Magnetic biochips: a new option for sensitive diagnostics. Journal of Magnetism and Magnetic Materials 293 (1):702-708.

Mihajlović, Goran, Peng Xiong, Stephan von Molnár, Keita Ohtani, Hideo Ohno, Mark Field, and Gerard J. Sullivan. 2005. Detection of single magnetic bead for biological applications using an InAs quantum-well micro-Hall sensor. Applied Physics Letters 87 (11): $112502 \% U$ http://link.aip.org/link/APPLAB/v87/i11/p112502/s1\&Agg=doi.

Miller, M. M., G. A. Prinz, S. F. Cheng, and S. Bounnak. 2002. Detection of a micron-sized magnetic sphere using a ring-shaped anisotropic magnetoresistance-based sensor: A model for a magnetoresistance-based biosensor. Applied Physics Letters 81 (12):2211 \%U http://link.aip.org/link/APPLAB/v81/i12/p2211/s1\&Agg=doi.

Miller, M. M., P. E. Sheehan, R. L. Edelstein, C. R. Tamanaha, L. Zhong, S. Bounnak, L. J. Whitman, and R. J. Colton. 2001. A DNA array sensor utilizing magnetic microbeads and magnetoelectronic detection. Journal of Magnetism and Magnetic Materials 225 (1-2):138-144 \%U http://www.sciencedirect.com/science/article/B6TJJ-42T4FB3S/2/6d15a99389fa27c041ea5c5ff6d88b30.

Moodera, J. S., Lisa R. Kinder, Terrilyn M. Wong, and R. Meservey. 1995. Large Magnetoresistance at Room Temperature in Ferromagnetic Thin Film Tunnel Junctions. Physical Review Letters 74 (16):3273 \%U http://link.aps.org/doi/10.1103/PhysRevLett.74.3273.

Morozov, Victor N., Stephanie Groves, Michael J. Turell, and Charles Bailey. 2007. Three Minutes-Long Electrophoretically Assisted Zeptomolar Microfluidic Immunoassay with Magnetic-Beads Detection. Journal of the American Chemical Society 129 (42):12628-12629 \%U http://dx.doi.org/10.1021/ja075069m.

Morozova, Tamara Y., and Victor N. Morozov. 2008. Force differentiation in recognition of cross-reactive antigens by magnetic beads. Analytical Biochemistry 374 (2):263-271 $\%$ http://www.sciencedirect.com/science/article/B6W9V-4R9JTXB2/2/29f23994f4d52579722ed41a9f21ee68.

Moskowitz, R., and R. E. Rosensweig. 1967. NONMECHANICAL TORQUE-DRIVEN FLOW OF A FERROMAGNETIC FLUID BY AN ELECTROMAGNETIC FIELD. Applied Physics Letters.

Mulvaney, S. P., C. L. Cole, M. D. Kniller, M. Malito, C. R. Tamanaha, J. C. Rife, M. W. Stanton, and L. J. Whitman. 2007. Rapid, femtomolar bioassays in complex matrices combining microfluidics and magnetoelectronics. Biosensors and Bioelectronics 23 (2): 191-200.

Mulvaney, S. P., C. N. Ibe, C. R. Tamanaha, and L. J. Whitman. 2009. Direct detection of genomic DNA with fluidic force discrimination assays. Analytical Biochemistry 392 (2):139-144 \%U http://www.sciencedirect.com/science/article/B6W9V-4WF4J575/2/d70c54d9fee98336fa2efdc517c9fa49.

Nam, J. M., K. J. Jang, and J. T. Groves. 2007. Detection of proteins using a colorimetric bio-barcode assay. Nature Protocols 2 (6):1438-1444.

Nam, J. M., S. J. Park, and C. A. Mirkin. 2002. Bio-barcodes based on oligonucleotidemodified nanoparticles. J. Am. Chem. Soc 124 (15):3820-3821.

Nam, J. M., S. I. Stoeva, and C. A. Mirkin. 2004. Bio-bar-code-based DNA detection with PCR-like sensitivity. J. Am. Chem. Soc 126 (19):5932-5933.

Nam, J. M., C. S. Thaxton, and C. A. Mirkin. 2003. Nanoparticle-based bio-bar codes for the ultrasensitive detection of proteins. Science 301 (5641):1884. 
Obata, K., H. Tajima, M. Yohda, and T. Matsunaga. 2002. Recent developments in laboratory automation using magnetic particles for genome analysis. pgs 3 (5):697708.

Oh, B. K., J. M. Nam, S. W. Lee, and C. A. Mirkin. 2006. A Fluorophore-Based Bio-Barcode Amplification Assay for Proteins. Small 2 (1):103-108.

Osterfeld, Sebastian J., Heng Yu, Richard S. Gaster, Stefano Caramuta, Liang Xu, Shu-Jen Han, Drew A. Hall, Robert J. Wilson, Shouheng Sun, Robert L. White, Ronald W.

Davis, Nader Pourmand, and Shan X. Wang. 2008. Multiplex protein assays based on real-time magnetic nanotag sensing. Proceedings of the National Academy of Sciences 105 (52):20637 -20640\%U http://www.pnas.org/content/105/52/20637.abstract.

Palecek, E., M. Fojta, and F. Jelen. 2002. New approaches in the development of DNA sensors: hybridization and electrochemical detection of DNA and RNA at two different surfaces. Bioelectrochemistry 56 (1-2):85-90 \%U http://www.sciencedirect.com/science/article/B6W72-454TMDK3/2/e41fec3453fd75f07a5bb024c2f45aaa.

Park, Sang Yoon, Hiroshi Handa, and Adarsh Sandhu. 2010. Magneto-Optical Biosensing Platform Based on Light Scattering from Self-Assembled Chains of Functionalized Rotating Magnetic Beads. Nano Letters 10 (2):446-451 \%U http://dx.doi.org/10.1021/n19030488.

Perez, J. Manuel, Lee Josephson, Terrence O'Loughlin, Dagmar Hogemann, and Ralph Weissleder. 2002. Magnetic relaxation switches capable of sensing molecular interactions. Nat Biotech 20 (8):816-820 \%U http://dx.doi.org/10.1038/nbt720.

Popplewell, J., R. E. Rosensweig, and R. J. Johnston. 1990. Magnetic field induced rotations in ferrofluids. Magnetics, IEEE Transactions on 26 (5):1852-1854.

Richardson, J., P. Hawkins, and R. Luxton. 2001. The use of coated paramagnetic particles as a physical label in a magneto-immunoassay. Biosensors and Bioelectronics 16 (912): 989-993\%U http://www.sciencedirect.com/science/article/B6TFC-447MWX3$1 \mathrm{~K} / 2 / \mathrm{f9a0cbcf3b2c406f0e6e0f875244 \textrm {e } 7 6 1 .}$

Richardson, J., A. Hill, R. Luxton, and P. Hawkins. 2001. A novel measuring system for the determination of paramagnetic particle labels for use in magneto-immunoassays. Biosensors and Bioelectronics 16 (9-12):1127-1132.

Rife, J. 2003. Design and performance of GMR sensors for the detection of magnetic microbeads in biosensors. Sensors and Actuators A: Physical 107 (3):209-218\%U http://apps.isiknowledge.com/full record.do?product=UA\&colname=WOS\&search m ode=CitingArticles\&qid=33\&SID=4Cae37FbIfNh@p1kGij\&page=26\&doc=252.

Romano, G., L. Sacconi, M. Capitanio, and F. S. Pavone. 2003. Force and torque measurements using magnetic micro beads for single molecule biophysics. Optics Communications 215 (4-6):323-331.

Safarik, I., and M. Safarikova. 2004. Magnetic techniques for the isolation and purification of proteins and peptides. BioMagnetic Research and Technology 2 (1):7.

Safarık, I., and M. Safarıkova. 1999. Use of magnetic techniques for the isolation of cells. Journal of Chromatography B 722:33-53.

Schotter, J. 2004. Comparison of a prototype magnetoresistive biosensor to standard fluorescent DNA detection. Biosensors and Bioelectronics 19 (10):1149-1156 \%U http://apps.isiknowledge.com/full record.do?product=UA\&colname=WOS\&search $\mathrm{m}$ ode=CitingArticles\&qid=33\&SID=4Cae37FbIfNh@p1kGij\&page=25\&doc=244.

Schrag, Deborah, Harinder S. Garewal, Harold J. Burstein, David J. Samson, Daniel D. Von Hoff, and Mark R. Somerfield. 2004. American Society of Clinical Oncology Technology Assessment: Chemotherapy Sensitivity and Resistance Assays. Journal of Clinical Oncology 22 (17):3631 -3638\%U http://jco.ascopubs.org/content/22/17/3631.abstract. 
Sheehan, Paul E., and Lloyd J. Whitman. 2005. Detection Limits for Nanoscale Biosensors. Nano Letters 5 (4):803-807 \%U http://dx.doi.org/10.1021/nl050298x.

Shelton, Daniel R., and Jeffrey S. Karns. 2001. Quantitative Detection of Escherichia coli O157 in Surface Waters by Using Immunomagnetic Electrochemiluminescence. Appl. Environ. Microbiol. 67 (7):2908-2915 \%U http://aem.asm.org/cgi/content/abstract/67/7/2908.

Shelton, W. A., K. D. Bonin, and T. G. Walker. 2005. Nonlinear motion of optically torqued nanorods. Physical Review E 71 (3):36204.

Shen, W., X. Liu, D. Mazumdar, and G. Xiao. 2005. In situ detection of single micron-sized magnetic beads using magnetic tunnel junction sensors. Applied Physics Letters $86: 253901$.

Shen, W., B. D. Schrag, M. J. Carter, J. Xie, C. Xu, S. Sun, and G. Xiao. 2008. Detection of DNA labeled with magnetic nanoparticles using MgO-based magnetic tunnel junction sensors. Journal of Applied Physics 103:07A306.

Shlyapnikov, Yury M., Elena A. Shlyapnikova, Tamara Ya Morozova, Igor P. Beletsky, and Victor N. Morozov. 2010. Detection of microarray-hybridized oligonucleotides with magnetic beads. Analytical Biochemistry 399 (1):125-131\%U http://www.sciencedirect.com/science/article/B6W9V-4Y1NV5X2/2/b47fc339d67aaa422184ee2f3b952ea9.

Speriosu, V. S., B. Dieny, P. Humbert, B. A. Gurney, and H. Lefakis. 1991. Nonoscillatory magnetoresistance in $\mathrm{Co} / \mathrm{Cu} / \mathrm{Co}$ layered structures with oscillatory coupling. Physical Review B 44 (10):5358-5361.

Stoeva, S. I., J. S. Lee, J. E. Smith, S. T. Rosen, and C. A. Mirkin. 2006. Multiplexed detection of protein cancer markers with biobarcoded nanoparticle probes. J. Am. Chem. Soc 128 (26): 8378-8379.

Stoeva, S. I., J. S. Lee, C. S. Thaxton, and C. A. Mirkin. 2006. Multiplexed DNA detection with biobarcoded nanoparticle probes. Angewandte Chemie 118 (20):3381-3384.

Strömberg, M., T. Zardán Gómez de la Torre, J. Göransson, K. Gunnarsson, M. Nilsson, M. Strømme, and P. Svedlindh. 2008. Microscopic mechanisms influencing the volume amplified magnetic nanobead detection assay. Biosensors and Bioelectronics 24 (4):696-703 \%U http://www.sciencedirect.com/science/article/B6TFC-4SXH9X23/2/afd6bade2a464e0861cf6ca86c94ec22.

Strömberg, Mattias, Jenny Göransson, Klas Gunnarsson, Mats Nilsson, Peter Svedlindh, and Maria Strømme. 2008. Sensitive Molecular Diagnostics Using Volume-Amplified Magnetic Nanobeads. Nano Letters 8 (3):816-821\%U http://dx.doi.org/10.1021/nl072760e.

Strömberg, Mattias, Teresa Zardán Gómez de la Torre, Jenny Goransson, Klas Gunnarsson, Mats Nilsson, Peter Svedlindh, and Maria Strømme. 2009. Multiplex Detection of DNA Sequences Using the Volume-Amplified Magnetic Nanobead Detection Assay. Analytical Chemistry 81 (9):3398-3406 \%U http://dx.doi.org/10.1021/ac900561r.

Tamanaha, C. R., S. P. Mulvaney, J. C. Rife, and L. J. Whitman. 2008. Magnetic labeling, detection, and system integration. Biosensors and Bioelectronics 24 (1):1-13.

Taton, T. A., C. A. Mirkin, and R. L. Letsinger. 2000. Scanometric DNA array detection with nanoparticle probes. Science 289 (5485): 1757.

Thomas, J., S. Kim, P. Hesketh, H. Halsall, and W. Heineman. 2004. Microbead-based electrochemical immunoassay with interdigitated array electrodes. Analytical Biochemistry 328 (2):113-122\%U http://apps.isiknowledge.com/CitedFullRecord.do?product=UA\&db id =WOS\&SID $=3 F$ 9k9AG37dGoJlJka@d\&search mode=CitedFullRecord\&isickref=134517330.

Tierno, Pietro, Josep Claret, Francesc Sagués, and Andrejs Cemacrbers. 2009. Overdamped dynamics of paramagnetic ellipsoids in a precessing magnetic field. Physical Review $E$ 79 (2):021501 \%U http://link.aps.org/doi/10.1103/PhysRevE.79.021501. 
Touhami, A., M. H. Jericho, and T. J. Beveridge. 2004. Atomic force microscopy of cell growth and division in Staphylococcus aureus. Journal of bacteriology 186 (11):3286.

Tsukamoto, A., K. Saitoh, D. Suzuki, N. Sugita, Y. Seki, A. Kandori, K. Tsukada, Y. Sugiura, S. Hamaoka, H. Kuma, N. Hamasaki, and K. Enpuku. 2005. Development of multisample biological immunoassay system using HTSSQUID and magnetic nanoparticles. Applied Superconductivity, IEEE Transactions on 15 (2):656-659.

Valberg, P. A., and J. P. Butler. 1987. Magnetic particle motions within living cells. Physical theory and techniques. Biophysical Journal 52 (4):537-550 \%U http://www.sciencedirect.com.proxy.lib.umich.edu/science/article/B94RW-4V8RST14/2/816c49353f7b5041cf43ff68109051c4.

Wang, J. 2003. Particle-based detection of DNA hybridization using electrochemical stripping measurements of an iron tracer. Analytica Chimica Acta 482 (2):149-155 \%U http://apps.isiknowledge.com/CitedFullRecord.do?product=UA\&db id=WOS\&SID $=4 \mathrm{~B}$ L5L1G2C26cdpkcA@9\&search mode=CitedFullRecord\&isickref=129260972.

Wang, Joseph, Guodong Liu, and M. Rasul Jan. 2004. Ultrasensitive Electrical Biosensing of Proteins and DNA: Carbon-Nanotube Derived Amplification of the Recognition and Transduction Events. Journal of the American Chemical Society 126 (10):3010-3011 \%U http://dx.doi.org/10.1021/ja031723w.

Wang, Joseph, Ronen Polsky, and Danke Xu. 2001. Silver-Enhanced Colloidal Gold Electrochemical Stripping Detection of DNA Hybridization. Langmuir 17 (19):5739$5741 \%$ http://dx.doi.org/10.1021/la011002f.

Wang, Joseph, Danke Xu, Abdel-Nasser Kawde, and Ronen Polsky. 2001. Metal Nanoparticle-Based Electrochemical Stripping Potentiometric Detection of DNA Hybridization. Analytical Chemistry 73 (22):5576-5581\%U http://apps.isiknowledge.com/CitedFullRecord. do?product=UA\&db id=WOS\&SID $=4 B$ L5L1G2C26cdpkcA@9\&search mode=CitedFullRecord\&isickref=122297636.

Wang, Joseph, Danke Xu, and Ronen Polsky. 2002. Magnetically-Induced Solid-State Electrochemical Detection of DNA Hybridization. Journal of the American Chemical Society 124 (16):4208-4209\%U http://dx.doi.org/10.1021/ja0255709.

Wang, S. X., S. Y. Bae, G. Li, S. Sun, R. L. White, J. T. Kemp, and C. D. Webb. 2005. Towards a magnetic microarray for sensitive diagnostics. Journal of Magnetism and Magnetic Materials 293 (1):731-736.

Wang, Zhixin, Xiaoli Wang, Shengquan Liu, Junfa Yin, and Hailin Wang. 2010. Fluorescently Imaged Particle Counting Immunoassay for Sensitive Detection of DNA Modifications. Analytical Chemistry 82 (23):9901-9908 \%U http://dx.doi.org/10.1021/ac102416f.

Weber, P. C., D. H. Ohlendorf, J. J. Wendoloski, and F. R. Salemme. 1989. Structural origins of high-affinity biotin binding to streptavidin. Science 243 (4887):85.

Weizmann, Yossi, Roey Elnathan, Oleg Lioubashevski, and Itamar Willner. 2005. Magnetomechanical Detection of the Specific Activities of Endonucleases by Cantilevers. Nano Letters 5 (4):741-744 \%U http://apps.isiknowledge.com/full record.do?product=UA\&colname=WOS\&search $\mathrm{m}$ ode=CitingArticles\&qid=45\&SID=4Cae37FbIfNh@p1kGij\&page=4\&doc=31.

Weizmann, Yossi, Fernando Patolsky, Eugenii Katz, and Itamar Willner. 2003. Amplified DNA Sensing and Immunosensing by the Rotation of Functional Magnetic Particles. Journal of the American Chemical Society 125 (12):3452-3454 \%U http://dx.doi.org/10.1021/ja028850x.

Weizmann, Yossi, Fernando Patolsky, Oleg Lioubashevski, and Itamar Willner. 2004. Magneto-Mechanical Detection of Nucleic Acids and Telomerase Activity in Cancer Cells. Journal of the American Chemical Society 126 (4):1073-1080 \%U http://dx.doi.org/10.1021/ja038257v. 
Wood, D. K., K. K. Ni, D. R. Schmidt, and A. N. Cleland. 2005. Submicron giant magnetoresistive sensors for biological applications. Sensors and Actuators $A$ : Physical 120 (1):1-6.

Yang, Liju, and Yanbin Li. 2006. Detection of viable Salmonella using microelectrode-based capacitance measurement coupled with immunomagnetic separation. Journal of Microbiological Methods 64 (1):9-16 \%U http://www.sciencedirect.com/science/article/B6T30-4G9GN1G6/2/5c1ff4f5094305f7af4d8776fc49f966.

Yellen, B. B., R. M. Erb, H. S. Son, R. Hewlin, H. Shang, and G. U. Lee. 2007. Traveling wave magnetophoresis for high resolution chip based separations. Lab on a Chip 7 (12):1681-1688.

Zardán Gómez de la Torre, Teresa, Mattias Strömberg, Camilla Russell, Jenny Göransson, Mats Nilsson, Peter Svedlindh, and Maria Strømme. 2010. Investigation of Immobilization of Functionalized Magnetic Nanobeads in Rolling Circle Amplified DNA Coils. The Journal of Physical Chemistry B 114 (10):3707-3713 \%U http://dx.doi.org/10.1021/jp911251k.

Zhang, Li, Jake J. Abbott, Lixin Dong, Bradley E. Kratochvil, Dominik Bell, and Bradley J. Nelson. 2009. Artificial bacterial flagella: Fabrication and magnetic control. Applied Physics Letters.

Zhu, Ningning, Aiping Zhang, Pingang He, and Yuzhi Fang. 2004. DNA Hybridization at Magnetic Nanoparticles with Electrochemical Stripping Detection. Electroanalysis 16 (23): $1925-1930 \% \mathrm{U}$ http://onlinelibrary.wiley.com/doi/10.1002/elan.200303028/pdf.

Zhu, Xiaoshan, Dayue Duan, and Nelson G. Publicover. 2010. Magnetic bead based assay for C-reactive protein using quantum-dot fluorescence labeling and immunoaffinity separation. The Analyst 135 (2):381\%U http://apps.isiknowledge.com/full record.do?product=UA\&colname=WOS\&search $\mathrm{m}$ ode=CitingArticles\&qid=23\&SID=4Cae37FbIfNh@p1kGij\&page =1\&doc=4.

Ziegler, Christiane. 2004. Cantilever-based biosensors. Analytical and Bioanalytical Chemistry 379 (7-8 \%U http://apps.isiknowledge.com/full record.do?product=UA\&colname=WOS\&search m ode=CitingArticles\&qid=33\&SID=4Cae37FbIfNh@p1kGij\&page=23\&doc=226). 